\title{
On state-of-charge determination for lithium-ion batteries
}

\author{
Zhe LI ${ }^{\mathrm{a}, \mathrm{b}}$, Jun HUANG ${ }^{\mathrm{a}}$, Bor Yann $\mathrm{LIAW}^{\mathrm{c}}$, Jianbo ZHANG ${ }^{\mathrm{a}, \mathrm{b} *}$
}

a State Key Laboratory of Automotive Safety and Energy, Department of Automotive Engineering, Tsinghua University, Beijing 100084, China

${ }^{b}$ Beijing Co-innovation Center for Electric Vehicles, Beijing Institute of Technology, Beijing 100081, China

${ }^{c}$ Energy Storage and Advanced Vehicles, Idaho National Laboratory, Idaho Falls, ID 83415, USA

*Corresponding authors: J. Zhang, jbzhang@mail.tsinghua.edu.cn; B.Y. Liaw,

Boryann.liaw@inl.gov. 


\begin{abstract}
Accurate estimation of state-of-charge (SOC) of a battery through its life remains challenging in battery research. Although improved precisions continue to be reported at times, almost all are based on regression methods empirically, while accuracy is often not properly addressed. Here, a comprehensive review is set to address such issues, from fundamental principles that are supposed to define SOC to methodologies to estimate SOC for practical use. It covers topics from calibration, regression (including modeling methods) to validation in terms of precision and accuracy. At the end, we intend to answer the following questions: 1) can SOC estimation be self-adaptive without bias? 2) Why Ah-counting is a necessity in almost all battery-model-assisted regression methods? 3) How to establish a consistent framework of coupling in multi-physics battery models? 4) To assess the accuracy in the SOC estimation, statistical methods should be employed to analyze factors that contribute to the uncertainty. We hope, through this proper discussion of the principles, accurate SOC estimation can be widely achieved.
\end{abstract}

\title{
Keywords
}

Li-ion battery; state of charge (SOC); calibration; regression; multi-physics battery model; statistical accuracy 


\section{INTRODUCTION}

The estimation of state-of-charge (SOC) of a battery is probably one of the most challenging topics in the battery research. SOC is the most critical state parameter, playing fundamental role in defining other state functions for proper functionality of the battery, viz. performance, reliability, and safety. To determine SOC reliably, precisely, and accurately is thus crucial. To date, SOC is still determined by empirical methods by capacity measurements, whereas its accuracy can hardly be validated in practical use, leaving reliability and safety issues of a battery too difficult to handle. Here, we intend to explain the fundamental principles in defining SOC and reveal the issues with SOC determination by empirical methods. Further discussions on attributes that contribute to uncertainty and difficulty in measurements, analytical derivations to correlate measurable parameters to SOC, regressions, and predictions constitute the entire review. We believe this is the first comprehensive review on this subject for the lithium-ion battery (LIB) that is quite overdue. We hope the discussions will help the practitioners in this field receive a better understanding of the meaning of SOC in different perspectives and the technical difficulties that they might encounter in the current practice. We also hope, by clarifying the ambiguity in defining and determining SOC in various methods, a more reliable and accurate SOC estimation can be achieved.

Earlier papers coining the term 'state of charge' date back to 1960s [1-3]. Yet, after more than half a century of continuous efforts, we are still debating the definition of SOC and struggling with finding a better way to determine SOC, as summarized in various recent reviews [4-10]. With progress made in electrification of vehicles (e.g. various types of electric vehicles or $\mathrm{xEVs}$ ) and grid energy storage systems, where large battery packs and banks with more than 
hundreds or thousands of batteries are being used, SOC estimation is even more critical, demanding and challenging. In these scenarios, battery systems rely heavily on reliable, precise, accurate SOC estimation to coordinate devices in the system in order to operate safely through seamless control and management of power inputs and outputs. Any weak link could easily cripple or even fail the entire system.

The SOC, different from other descriptors such as current and voltage that can be measured on board directly in most situations, is a subtle descriptor for a battery system since it is not easily and directly measurable. A number of reasons attribute to this difficulty, originating from both thermodynamics and kinetics (more details shall be discussed in Section 3). In essence, a true SOC should be the one defined by principles governed by thermodynamic equilibrium; yet, in reality, what we want in practical applications is a simple descriptor that tells the availability of usable energy and capacity in the system that can be deployed to conduct work under polarization, in which SOC is expected to be a function of numerous factors governed by the kinetic regimes with all sorts of electrochemical, mechanical and thermal fields and gradients. Worst off, these factors are often coupled, creating complicated transmuting results that make the empirical methods that rely on correlations ambiguous and difficult to be defined for the SOC determination.

To facilitate further discussions on this subject, we shall call the SOC that is governed by the thermodynamic equilibrium the "true SOC" or " $t-S O C$," whereas the SOC that is determined empirically through engineering methodologies to service the purposes for any functions in a battery system "empirical SOC" or "e-SOC." In most places, such distinctions might not be necessary, and we shall just use generic "SOC" to express the concept that is widely used in 
the literature to date.

It is also worth noting that, in the literature, most of researchers studying on this subject often emphasize precision (in respect to $e-S O C$ ), much less on accuracy (especially with regard to $t-S O C)$, as to how to perform a better $e-S O C$ estimation. Such an $e-S O C$ estimation is mainly accomplished by counting the electrical charges in and out of the batteries (i.e. the capacity in coulombs or Ah) in a typical coulomb-counting (or Ah-counting) technique as expressed by Eq. (1):

$$
\operatorname{SOC}(t)=\operatorname{SOC}_{0}-\frac{\int_{0}^{t} \eta i d \tau}{Q_{\text {rated }}}
$$

where $\mathrm{SOC}_{0}$ represents the initial SOC; $Q_{\text {rated }}$ the rated capacity under a certain specification by the manufacturer; $\eta$ the coulombic efficiency; $i$ the current at any instance of time, which is by convention negative at charge and positive at discharge. One should pay attention to the distinction between precision and accuracy with regard to SOC estimation. There are many genuine approaches reported in the literature today that could precisely determine $e$-SOCs to serve the engineering needs, but often these $e$-SOCs so-determined were not validated with "t-SOCs" to determine their accuracy. This aspect is particularly important but difficult and vulnerable when the battery ages and capacity fades. Such disparities could cause serious concerns in reliability, durability and safety issues in practical applications.

Although it may be ostensibly easy and likely accountable to find electrical energy balance (in term of charge and voltage) quickly in a battery system, the reality behind the balance of energy is much more complicated than the electrical one alone. Such an energy balance should include chemical (e.g. via mass transfer and reaction), thermal (e.g. via heat 
generation, retention, flow, and exchange with the environment), and mechanical ones (e.g. via volume changes and micromechanical forces-induced stress and strain changes); and, each and every may in turn affect the result of the electrical energy balance over a significant range of spatial and temporal scales. The complicated situation constitutes the very reason why it is so difficult to determine SOC reliably, precisely, and accurately.

Disregarding the complexity in the energy balance in the determination of battery state functions, the Ah-counting method to obtain SOC remains a practical solution used by the majority in the battery technology industry [10]. The problems of the Ah-counting method include: (1) uncertainty and inherent errors in the determination of the initial $\mathrm{SOC}_{0}$, (2) the accumulative errors over a long period of time due to the imprecision introduced by the time counters and current measuring transducers, as well as coulombic efficiency; (3) the use of rated capacity $Q_{\text {rated }}$ as the denominator in the SOC calculation, since $Q_{\text {rated }}$ is determined more or less arbitrarily by test protocol and procedure used by the cell manufacturers or device OEMs, and (4) systematic errors introduced by the tests. To overcome these problems, there are several solutions that have been suggested in the past to improve precision in the measurements, to validate the results against other assessments of information for the sake of the accuracy, to correct possible sources of errors, and to reduce the impacts of the errors on the method of SOC estimation. For instance, in principle the SOC should have a certain unique correlation with other measureable state parameters, such as the open-circuit voltage $\left(\mathrm{OCV}\right.$ or $\left.V_{\mathrm{OC}}\right)$ or the electrochemical impedance in a specific frequency range [4,5]. These correlations can be pre-determined in the labs and made into look-up tables to pinpoint or calibrate the SOC values. To improve precision, one should strive to diminish any deviations 
from the current integration and technique, via better hardware platforms and instruments or intelligent software-based regression methods, most of which are battery-model-assisted data deduction [5].

Using suitable battery models and regression techniques has been touted as a powerful approach to achieve viable results. Therefore, a significant level of efforts has been engaged in the past targeting on building accurate models. These efforts include those using various types of multi-physics framework to couple principal system properties, such as those governed by electrochemical and thermal processes; utilizing simplified mechanism descriptions, dimensional reduction, and efficient parameter identification; and, engaging sufficient validation of model precision and accuracy. A typical exercise in most regression methods from modern control theory, including those using least square [11-14], filters [15-20], estimators [8], artificial neural networks [21-26], genetic algorithms [27,28], and so on, demonstrates improved precision. However, by and large, without any adequate validation with SOC values determined separately from reliable techniques, such as OCVs measured after equilibrium, the accuracy remains undetermined. This is an important aspect that future work should strive to pay attention to and address properly.

Even if a battery model were precise and accurate enough to describe specific features of a single cell of a specific geometry and size, to enable such a model applicable to cells of different geometries and sizes is not trivial. This is conceivable based on the implications of the energy balance issues, as explained earlier. These issues depend on spatial and temporal factors that interact incongruously. It is therefore not straightforward to scale up parameters in the model with extended physical dimensions or time. Likewise, to apply the same model 
to simulate behavior of a battery pack, additional issues such as cell-to-cell variations may still get in the way to affect precision or accuracy of the prediction or estimation. This issue has made the SOC estimation a statistical practice beyond the conventional wisdom to believe that the SOC could be definitively determined with sufficient precision and accuracy in reality. It is important to point out here that the $t-S O C$ as governed by the thermodynamic equilibrium is supposed irrelevant to temporal and spatial variations, whereas the $e$-SOC in the kinetic regime could be sensitively affected by such variations.

Here, we shall begin with the explanations of fundamental concept and underlying principles that govern battery state functions and review SOC-related subjects with relevant discussions on issues encountered. Although in early work on the SOC estimation of Ni-Cd batteries [3], using OCV, terminal cell voltage, and impedance at certain frequencies has been mentioned as possible SOC estimation methods, how to apply these methods in lithium-ion batteries, for example, might not be as trivial as that has been suggested. Therefore, in this work, we shall survey the landscape of relevant SOC issues and provide a forum for unified discussions on the subjects. The hierarchy of discussions on the topics shall follow a logic tree, as outlined in Figure 1. 


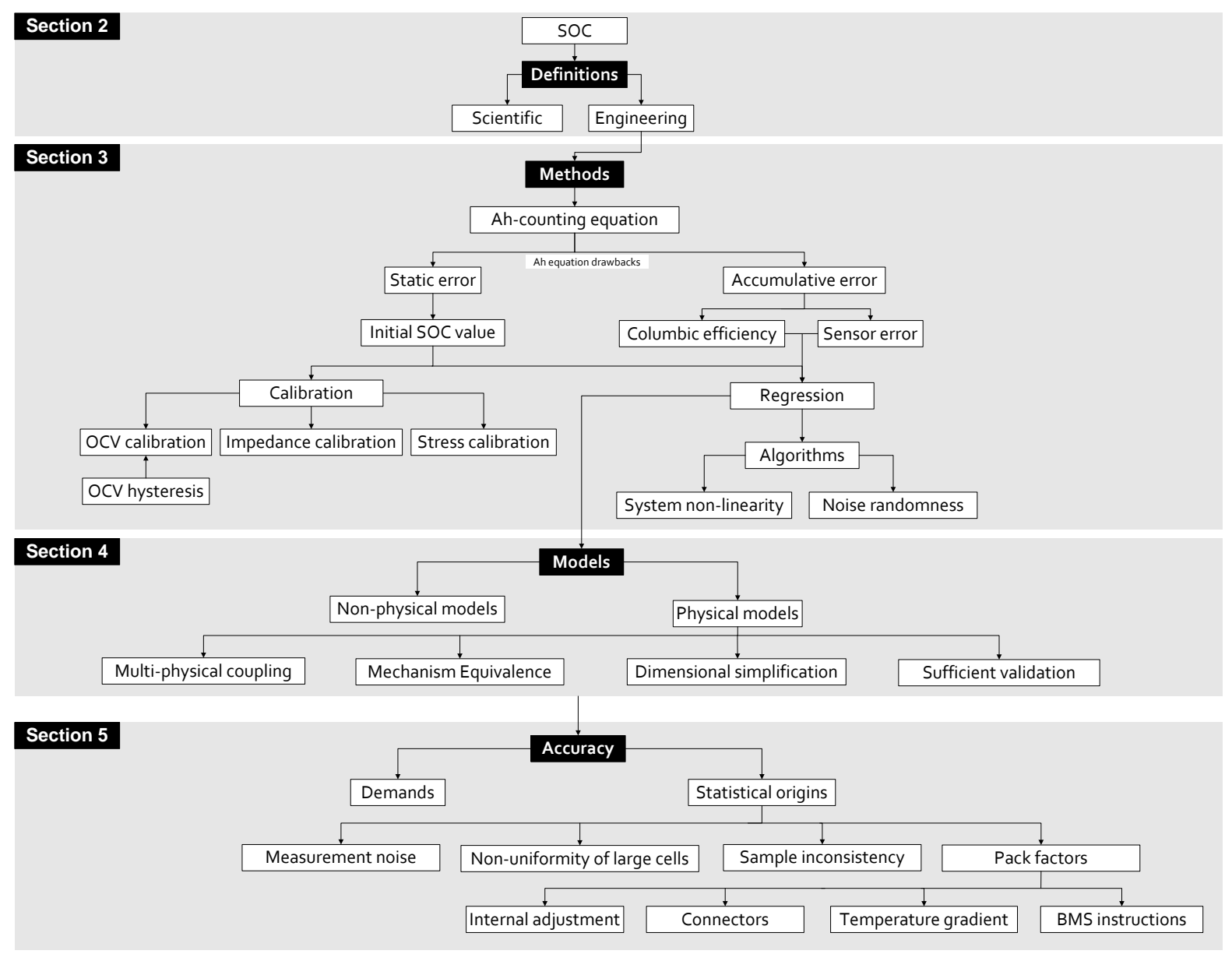

[Figure 1]

\section{HOW TO DEFINE SOC}

To fully capture the essence of state functions, such as SOC, we shall start from the basic understanding of SOC from materials level to the system. Without such an understanding, it is hard to establish the basis for SOC estimation through model simulations, approximations, calculations and validations. 


\subsection{Definition from thermodynamics}

\subsubsection{SOC in an electrode}

The SOC is a state function of a battery system. To abide by the laws of thermodynamics, the state of a chemical system is defined only when, at constant temperature and pressure, the species in the system are in equilibrium. At such a condition, the composition of the active material of an electrode is unique, and so is the concentration of the electrochemically active species in that composition. Accordingly, the Gibbs free energy of formation associated with this composition is thus defined. In other words, the electrode potential of this composition is unique against a reference potential. For a lithium-ion battery, it implies that the lithium content in the electrode, say $x$ in $\operatorname{Li}_{x} M_{y} O_{z}$, is fixed at such a condition. The potential of this composition, according to the Gibbs free energy of formation, is constant versus a reference electrode such as a Li metal (often expressed as $\mathrm{Li} / \mathrm{Li}^{+}$):

$$
\begin{aligned}
& E=-\frac{\Delta G\left(\mathrm{Li}_{\mathrm{x}} \mathrm{M}_{\mathrm{y}} \mathrm{O}_{\mathrm{z}}\right)}{n F} \\
& \quad=\text { a unique, characteristic potential (versus } \mathrm{Li} / \mathrm{Li}^{+} \text {), }
\end{aligned}
$$

where $n$ is the number of electrons involved in the charge transfer per unit formula, and $F$ the Faraday constant. This expression is consistent with one expected from the Nernst equation:

$$
E=-\frac{R T \ln \left(a_{\mathrm{Li}}\right)}{n F}=\text { a unique, characteristic potential (versus } \mathrm{Li} / \mathrm{Li}^{+} \text {), }
$$

in which the activity of $\mathrm{Li}, a_{\mathrm{Li}}$, is fixed. In this case, SOC is defined as,

$$
S O C=f\left(c_{S}\right)
$$

here $c_{s}$ is the lithium content in the active material (i.e. $\mathrm{x}$ in $\mathrm{Li}_{\mathrm{x}} \mathrm{M}_{\mathrm{y}} \mathrm{O}_{\mathrm{z}}$ ) with a specific value in 
correspondence with $a_{\mathrm{Li}}$.

\subsubsection{SOC in a single cell}

A lithium-ion cell comprises two electrodes, the positive (cathode in a galvanic cell) and the negative (anode). The cell voltage reflects the difference in the potentials of the two. When the two electrodes are in equilibrium, each stays at specific potential and lithium content in the crystal structure, say $c_{S}^{c}$ and $c_{S}^{a}$, respectively. Here, the SOC could be expressed by a concentration vector, $\vec{c}_{s}=\left(c_{s}^{c}, c_{s}^{a}\right)$. However, two scenarios spoil the feasibility of using the concentration vector to define the SOC for a cell: (1) the lithium content in solids or the electrolyte can hardly be measured directly in experiments or in a real cell <1>; and (2) a simple numerical indicator is more desirable than a vector with two concentration values. Therefore, it is easier to turn to the classical framework using OCV [29-31], a single-valued parameter that can be measured at equilibrium, which expresses the difference in lithium-ion concentrations in the electrodes. The correlation between the OCV and concentrations can be simplified as follows:

$$
\mathrm{OCV}=f_{c}\left(c_{s}^{c}, c_{e}^{c}\right)-f_{a}\left(c_{s}^{a}, c_{e}^{a}\right)
$$

where $f_{c}$ and $f_{a}$ refer to the OCV vs. SOC curves of the positive and negative electrode,

$<1>$ A neutron radiography or neutron imaging method using neutron sources with specific energy has been suggested for in situ and real-time detection of the total concentration of $\mathrm{Li}$ ions in certain volume, whereas a similar method named neutron depth profiling may also have a high resolution in the vertical direction (depth) of the volume, thus can accurately locate the ion concentration at a position in the three-dimensional volume. 
respectively, as a function of lithium-ion concentration in each electrode. Here, $c_{e}^{c}$ and $c_{e}^{a}$ are the lithium-ion concentrations in the electrolyte phase adjacent to the cathode and anode, respectively. At equilibrium, the lithium-ion concentration in the electrolyte is uniform in the cell, i.e. $c_{e}^{c}=c_{e}^{a}$. Therefore, the lithium-ion concentration in the electrolyte is irrelevant to the SOC at each electrode. Hence, the OCV is exclusively determined by $c_{s}^{c}$ and $c_{S}^{a}$, as one would expect from the Nernst equation,

$$
\text { OCV }=f_{\text {cell }}\left(c_{s}^{c}, c_{s}^{a}\right)=f_{\text {cell }}\left(\vec{c}_{s}\right)
$$

If the inverse function exists for Eq. (6),

$$
\vec{c}_{s}=f_{\text {cell }}^{-1}(O C V),
$$

one can use the OCV to infer the SOC of the cell. Therefore, if and only if the correlation between SOC and OCV is one-to-one, as the electrochemical equilibrium governs, we can use the OCV to infer the SOC of the cell as follows:

$$
S O C=f_{\text {cell }}^{-1}(O C V)
$$

In general, the OCV vs. SOC curve can be determined by techniques such as galvanostatic intermittent titration technique (GITT) [32]. For convenience, an approximation of this curve with sufficient precision can be constructed by averaging the $\mathrm{C} / 25$ charge and discharge curves, as suggested in Refs. [29-31].

\subsection{Definitions from engineering perspective}

\subsubsection{Engineering approaches}

The definition of SOC from thermodynamic perspective presents a fundamental framework 
to depict the processes that determine the state of a battery cell. Nonetheless, applying this basic concept in real applications is practically infeasible. An expedient approach is needed to serve engineering purposes, such as estimating remaining capacity in a cell or battery system. Taking the powertrain as an example, there is a preset operating window that delineates the operation regime for the battery system. Battery performance needs to satisfy such power and energy demands to fulfill the functionality as an "engine" in the powertrain. Battery performance characteristics need to be defined as a function of battery's SOC. This state function needs to be tracked during an operation (often in so-called "duty schedule" or "duty cycle") to monitor the conditions of the battery system and to project its ability and capability to perform certain functions in a mission. This requirement is very different from the SOC defined at equilibrium. In other words, what is useful and critical in practical applications is the projection of a battery's transient capability in a duty cycle to a descriptor that can be easily accessible. The definition of SOC in such an engineering practice is thus different from the one defined in thermodynamic conditions. One of the most widely accepted conventions is the one given in the USABC Battery Test Procedure Manual [33] as follows:

$$
S O C=\frac{Q_{\text {res }}}{Q_{\text {rated }}} \times 100 \%
$$

where $Q_{\text {res }}$ represents the remaining capacity in a battery under a given testing condition, and $Q_{\text {rated }}$ the rated capacity under the same specific condition. In principle, both $Q_{\text {rated }}$ and $Q_{\text {res }}$ should be quantifiable, if Ah counting has sufficient precision and accuracy in a duty cycle.

The most troubling issue with this convention is the challenge in quantifying $Q_{\text {res }}$ and $Q_{\text {rated }}$ precisely and accurately in practical applications, especially as the battery or the system ages. On one hand, to project $Q_{\text {res }}$ accurately in a duty cycle is intrinsically challenging, because a 
truthful $Q_{\text {res }}$ projection is difficult due to the fact that its variations are "load-specific" in the cycle. On the other hand, $Q_{\text {rated }}$ is a quantity determined from laboratory testing for a specific cell design. Due to cell variability, $Q_{\text {rated }}$ is not truly constant from cell to cell in reality. Its accuracy depends on various attributes, including cell-to-cell variation from manufacturing quality, precision of the test equipment, errors created from test protocols and conditions, and quality control of the testing environment. Furthermore, since the operating conditions in real world are more complex than those used in the test procedure, the validity of using $Q_{\text {rated }}$ for SOC estimation as suggested by Eq. (9) is thus becoming questionable, not to mention the implications from cell-to-cell variations and aging-induced issues.

To mitigate these difficulties, a few suggestions for improvements have been proposed recently to modify the basis for SOC calculation. For instance, a more accommodating and responsive method that can accommodate possible capacity variations has been proposed [29-31]. In this method, the basis for the SOC calculation is the "maximum capacity" $\left(Q_{\max }\right)$, as determined in the test at a very low rate (such as $\mathrm{C} / 25$ or a rate beyond which the capacity does not increase noticeably anymore with rate) for a cell, as follows:

$$
S O C=\frac{Q_{\mathrm{res}}}{Q_{\max }} \times 100 \%
$$

Accordingly, the $Q_{\max }$ refers to the optimal capacity that an individual cell is supposed to deliver under minimal kinetic effects, and it should be close to the thermodynamic limit. In this practice, the SOC so calculated would be very close to that determined by GITT or other reliable means for calibration and validation to retain precision and accuracy [29]. 


\subsubsection{Practices in engineering definitions}

Using engineering approaches to define SOC such as in Eq. (9) and (10) it mostly relies on capacity calculation, inevitably introducing kinetic features into these methods. To determine SOC for engineering purposes, the following practices should be observed:

(1) From the user's perspective: Battery SOC is defined as $0 \%$ when the users can no longer extract any capacity (disregarding possible energy recovery after a short rest). Meanwhile, the SOC is defined as $100 \%$ when the battery is fully recharged (e.g. usually using a charging process with a two-stage constant-current, constant-voltage (CC-CV) profile to realize a more efficient charging). The SOC definition with this principle shall ensure that the user observes a full SOC range on the dashboard and have a better user experience.

(2) To estimate SOC or validate SOC estimation, the factors impacting the kinetics should all be predetermined and followed in the entire estimation process, including temperature and current (or rate) variations.

(3) If using the Ah-counting method (see Eq. (1)), the coulombic efficiency and the $Q_{\text {rated }}$ at the denominator should both be calibrated under the predetermined kinetic factors in the above practice.

(4) If a battery model is assisting the estimation method, the parameters of the model, such as the OCV-SOC curve and the circuit elements of the battery in the equivalent circuit model (ECM); should also be calibrated along with the predetermined kinetic factors. For instance, the studies on entropy coefficient of a battery cell showed that the OCV-SOC curve is rather insensitive to temperature [34]. The sensitivity of the parameters to the accuracy of the model prediction should be well characterized and understood. 
The kinetic characteristics that associate with $e-S O C$ is the origin of many complex issues of SOC estimation: they contribute largely to the errors of Ah-counting method and also bring so much trouble to the task of yielding accurate parameterization of battery models. On the contrary, proper understanding and handling of these kinetic features should make it possible to trace the SOC fluctuations by tracking important external variables. Otherwise, to estimate SOC using thermodynamic definition as depicted in Section 2.1 is impractical. This tracing technique is traditionally accomplished by the Ah-counting method. Therefore, to gain better insight how to accomplish and perfect this tracing technique we should dissect its drawbacks in order to provide better solutions. This aspect will be analyzed in detail in Section 3.

\section{METHODOLOGIES FOR SOC DETERMINATION}

The Ah-counting method is the most commonly used approach for SOC determination in practical applications. The principle of this method is rather straightforward: by knowing $Q_{\text {rated }}$ and the capacity that has been dispensed, one can estimate the maximum capacity remains in the battery, as delineated in Eq. (1). Since both current and time can be measured conveniently, this simple method provides the most practical tool for tracking the changes in SOC. However, this method is prone to errors: the error related to initial SOC determination; the accumulative errors from sensing instrumentation during the measurement of current and time; the variations in actual $Q_{\text {rated }}$ introduced by cell variability and capacity fade; and the uncertainty in coulombic efficiency over aging. To alleviate the uncertainties induced from these errors, a number of improvements and approaches have been suggested to date. 


\subsection{Calibration methods}

To minimize the SOC inaccuracy by the Ah-counting method, a logical solution is to perform calibration, a procedure often carried out by correlating the SOC with variables, such as OCV, impedance at certain frequencies, or surface stress when the battery is compressed by rigid plates with a constant pressure on both sides of the cell, to name a few.

\subsubsection{The correlation between SOC and OCV}

Attempts to correlate SOC with OCV could date back to 1960s by the work of Latner [3]. As explained in Section 2.1.1, this relationship of SOC and OCV is definitive as suggested by the thermodynamic principle when equilibrium is achieved. However, in reality some difficulties remain as barriers to yield this relationship accurately, if the governing principles could not be or were not properly observed in the experiments and analyses. Here, a few examples shall illustrate such aspects.

\subsubsection{From active material aspects}

Encountering of voltage plateaus - For some active materials such as lithium iron phosphate (LFP) the OCV vs. SOC curve exhibits a flat voltage plateau over the useful SOC range, and when coupled with a negative electrode such as graphite, a few voltage plateaus still exist in certain SOC ranges. The experimental results from a common LFP battery showed that the battery OCV may only vary $<5 \mathrm{mV}$ over a span of $15 \%$ SOC [35]. In this respect, using OCV to calibrate the SOC value needs high precision voltage measurements, most likely infeasible in practical applications with low-cost transducers. 


\subsubsection{Rest interval for equilibration}

It is prerequisite that only the OCV measured at equilibrium defines $t-S O C$, which could then be used to calibrate the $e$-SOC estimated by an engineering approach. Thus, to allow the cell to rest in order to determine its stable OCV over a sufficient (but non-excessive) period of time is essential. However, a legitimate rest period is also hard to determine, since it depends on the conditions before and during the rest. In other words, the type and magnitude of the perturbation introduced to the system prior to the rest could be a dominant factor in setting the duration to equilibrate. Such information is often unattended in practical applications to guide an accurate SOC determination. Inaccuracy incurred while the OCV is still in transient affects the fidelity of the calibration. Analogously, if the rest is excessive, self-discharge may occur and undermine the accuracy as well. In principle, the rest duration could be affected by temperature, SOC and the type and extent of prior perturbation. A reasonable rest interval is typically in the range of an hour to several days [36-40]. In a well-controlled experimental environment, using a protocol to decipher voltage variations within a preset precision limit and a reasonable time step in the data acquisition routine, say $<2 \sigma$ of a transducer's resolution within 20 min of an interval, might give a satisfactory result.

\subsubsection{Temperature dependence}

In general, the OCV vs. SOC curves over a limited temperature range are very similar within the operating conditions (e.g. from $-20^{\circ} \mathrm{C}$ to $60^{\circ} \mathrm{C}$ ), but they are still temperature-dependent, as expected from the entropy contribution; thus, such a dependence as expressed in $d V_{O C} / d \mathrm{~T}$ is largely the entropy changes in the battery system, depicted by the entropy coefficient, $d \Delta \mathrm{S} / d \mathrm{~T}$, which can be derived from the following: 


$$
d V_{O C} / d \mathrm{~T}=-1 / n F(d \Delta \mathrm{G} / d \mathrm{~T})=-1 / n F(d \Delta \mathrm{H} / d \mathrm{~T})+\mathrm{T} / n F(d \Delta \mathrm{S} / d \mathrm{~T})
$$

Since $d \Delta \mathrm{H} / d \mathrm{~T}$ is usually negligible, $d V_{O C} / d \mathrm{~T}$ is thus primarily attributed to $d \Delta \mathrm{S} / d \mathrm{~T}$.

Experimental results showed that the value of the entropy coefficient is dependent on the SOC (in reference to a specific composition of the active material) and temperature range, but generally it is small. For example, a temperature change of $10^{\circ} \mathrm{C}$ may introduce a variation in OCV on the order of $3 \mathrm{mV}$ for a cell using layered $\mathrm{Li}_{x} \mathrm{Ni}_{1 / 3} \mathrm{Mn}_{1 / 3} \mathrm{Co}_{1 / 3} \mathrm{O}_{2}$ and $\mathrm{Li}_{\mathrm{x}} \mathrm{Mn}_{2} \mathrm{O}_{4}$ spinel blended cathode, as reported in Ref. [34].

\subsubsection{Hysteresis induced by phase transition}

The impact of hysteresis has also been reported in the past [36,41-48], which could affect the intricate correlation between OCV and SOC. Here, the hysteresis means that the electrode potential exhibits a disparity, which is path-dependent during a charge-discharge cycle, at the same composition. Sometimes the potential differences could reach as large as tens of $\mathrm{mV}$, making the reproducibility of OCV uncertain for the SOC calibration. Some experimental results depicting the characteristics along with possible mechanistic models to explain the hysteresis phenomena are summarized in Table 1 and Table 2.

Even though the hysteresis is a concern, especially for materials that have extended voltage plateaus as in $\mathrm{Li}_{x} \mathrm{FePO}_{4}$-based systems, so far the magnitude of the impact on the calibration remains small and does not cause much concern in the SOC estimation.

\subsubsection{The correlation between SOC and impedance}

Besides using OCV to calibrate SOC, the impedance of battery has been suggested for such an application. In principle, using impedance to calibrate SOC is very challenging mostly due 
to the complexity of resolving various impedance attributes and allocating their contributions to the spectrum obtained by electrochemical impedance spectroscopy (EIS). It is important to point out that the equivalent circuit diagram (ECD) used in the data analysis fitting usually does not explicitly display a one-to-one mechanistic correspondence to the physical processes in the actual battery reactions. For example, a typical ECD does not separate attributes from positive and negative electrodes. Nor does it explicitly separate attributes in a process such as charge transfer, in which different mechanisms of mass transport (e.g. lithium ion transport in the liquid pore channels of the electrode and in the solid grains) could affect the kinetics of the charge transfer at the electrode-electrolyte interface. Therefore, the fitting in the EIS data analysis with an ECD could remain ambiguous with respect to mechanistic understanding of the kinetic effects on SOC projection, and this uncertainty casts a very challenging task for the calibration. Using impedance to calibrate the SOC has been proposed in the literature [4,5], and its drawbacks are listed in Table 3.

\subsubsection{The correlation between SOC and mechanical stress}

Using mechanical stress exhibited on the battery surface for SOC calibration is another concept reported recently [49]. The experimental data for the correlation between SOC and mechanical stress is usually obtained by measurements with a setup in which the battery is compressed by two rigid plates clamped onto two parallel sides of its containment. A pressure transducer is affixed on one of the surfaces. The principle of this approach is based on an assumption that the thickness or volume change of a battery in the free state is in proportion 
to the surface stress under a compressed state $\langle 2\rangle$ : when the SOC changes, the battery's dilation and contraction shall be reflected in the changes in thickness of the electrodes, inducing the variations of the surface pressure (force on the rigid plates). The correlation between the surface pressure and SOC is assumed independent of the temperature and current rate [49]. However, in our opinion, the methods of employing mechanical stress for SOC calibration need further improvements of the techniques and better understandings of the underlying mechanism in order to assure their validity. At the present time, the number of publications with evidence that could support corroborative arguments is few. Nonetheless, this approach offers potential for online monitoring and room for improvements, if some key issues listed in Table 4 could be resolved.

\subsubsection{Effect of aging and degradation}

Battery aging and degradation add another layer of complexity in the calibration: since all aforementioned factors such as OCV, impedance, and mechanical stress are all affected by

$<2>$ The measurement of stress evolution is seemingly more convenient than that of thickness or volume change. The thickness measurements of cells in a free state often require a strain or displacement transducer, e.g. a strain gauge (such as digital micrometer or linear variable differential transformer, LVDT), or imaging equipment (e.g. high-resolution optical/laser microscope or X-ray based techniques such as computerized axial tomography (or CT scan). The volume change is often measured by means of three-dimensional imaging devices or methods based on Archimedes' principle. Most of these measurements require support of special tools or devices. In each method, there are precision issues with instrumentation and the process, influences by external factors such as surface roughness, uniformity, mechanical distortion (bending, twisting, etc.), or uncertainty and error caused by shape, dimensional scale or aspect ratio changes in the cell that are not reversible. 
aging and degradation, as often reflected in the SOH of the battery. When a cell went through a series of aging and cycling conditions, the correlations of these factors with SOC all require re-calibration. For instance, the $V_{O C}=f(\mathrm{SOC})$ curve of a cell could be affected by aging and degradation, whereas the complicated $\mathrm{SOH}$ estimation is another challenge that also depends on aging conditions which is scarcely discussed in the literature so far. However, recent work $[30,31,50,51]$ indicated that such complicated relationship could be analyzed, quantified, and projected to derive the variations in the $V_{O C}=f(\mathrm{SOC})$ curve as a function of aging condition. An incremental capacity (dQ/dV) analysis and a mechanistic model were used to incorporate degradation modes that account for either thermodynamic attributes such as active materials loss in the electrolyte and electrodes or kinetic attributes such as impedance changes into the assessment of impacts from aging on OCV-SOC relationship and SOC estimation.

\subsection{Regression methods}

The application of calibration methods to ensure the validity of SOC estimation is limited in practice mainly because all these correlations based on OCV, impedance, or stress with SOC require an equilibrium condition to warrant reliable readings from the measurements, which can only be guaranteed when the battery has had a sufficient rest.

To enable practical applications, other possible solutions have been actively pursued by many researchers. Some have suggested including the use of high-precision sensors or transducers (although sharp increases in costs [10] could be a concern) and more in-depth investigations into the factors that impact $Q_{\text {rated }}($ the denominator in Eq. (1)). In the literature, it is generally accepted that, to determine $Q_{\text {rated }}$ under a predetermined cut-off voltage accurately, important 
factors should include ambient temperature, $\mathrm{SOH}$ (or often represented by cell resistance) and current (or rate) [50,51]. From the perspective of energy conservation, these factors should include all that affect the energy balance and efficiency in the battery system, including those leading to losses due to thermal (heat dissipation) or mechanical (hysteresis) origins.

In practical applications, costs of high-precision sensors or transducers and complicated setup to afford conditions to achieve accurate $Q_{\text {rated }}$ measurements could become prohibitive for implementation. Thus, alternative solutions are desired. Most of the recent efforts consider regression techniques the most feasible.

The most accepted regression methods are Kalman filter (KF)-based and its derivatives. The KF method is a recursive process in which each step reallocates a trust weight of two sources of outcomes as recursive inputs until a finite solution is reached within a set of criteria [15-20]. One source is the SOC value estimated from the Ah-counting method, and the other estimated from a state-space model. For batteries, the state-space model is formulated often using differential equations describing the relations between SOC and other measurable variables, including at least terminal voltage, $\mathrm{OCV}$, and current. The weight in each recursive step is set by reliability criteria of the state-space model represented by an intermediate parameter known as 'innovation,' which is the difference between the one (e.g. the terminal voltage in most examples) estimated by the state-space model and the measured value. If this innovation is sufficiently small, the joint estimated result should basically equal to the one sourced from the Ah-counting. Otherwise, the result from Ah-counting would be corrected by adding a supplementary item obtained by multiplying the innovation with the Kalman gain (KG) to take into account the source from the state-space model. 
The basics illustrated by the KF-based methods help to summarize the building blocks in most of the regression methods, which include:

- A battery model, i.e. the state-space model, to describe the correlation between SOC and measurable variables in the system.

- An algorithm, which is used to reasonably incorporate different sources or to distinguish the key indexes among all sources.

An accurate battery state-space model is theoretically difficult to establish. Although in the literature a handful of relevant efforts have been reported on how to construct this model more accurate, the true characteristics of a reliable model for accurate SOC estimation are still not fully identified. For example, 'multi-physics' models are frequently used by many that are interested in this subject. These models rely on accurate parameterization to make the model reliable. However, the strong coupling among key parameters remains as a challenging task for the modelers attempting to increase the models' fidelity with reality. In Section 4, we shall go into details about modeling and its relevant issues.

Discussions on algorithm-related issues are the most abundant in the literature that comes with a long history of study. In Section 3.3, we shall review the evolution in the research on algorithms used for SOC estimation.

Finally, for most of the regression methods, the Ah-counting process is always part of the process in the regression. In Section 3.4.2, we shall touch the basis on why Ah-counting is present in most of the SOC estimation methods. Is it feasible to fulfill the goals of the SOC estimation with sufficient precision only from the state-space models? Clarifying this issue may provide a basis for developing a better SOC estimation. 


\subsection{Regression algorithms}

\subsubsection{Algorithm is popular}

According to published papers from the beginning of 2014 to March 2015, we conducted an analysis on the sub-topics of a distribution of SOC-related publications. The literature search retrieved 53 papers $\langle 3\rangle$, which were narrowed down to 50 manually after removing three that are of little relevance. Among them 23 papers are related to the improvement of algorithms, whereas battery model-related papers are seven (excluding OCV models). In recent years, the algorithmic studies have occupied half of the SOC-related research activities, and special attention has been paid to improve accuracy of SOC estimation by algorithm improvements. The trend indicates that the interests on SOC-related research are shifting from those on materials and electrochemistry to those on system control, diagnoses, data mining and analyses using applied mathematics.

\subsubsection{Algorithm evolution}

Lots of different algorithms can be used to assist the regression of SOC, such as least square (LS) (e.g. [11-14]), Kalman filter (KF), extended KF (EKF) (e.g. [15-17]), sigma point KF

<3> Search using title:(batter*) AND ("state of charge" OR "capacity estimation" OR "SOC") on Web of Science core journals resulted in a collection of SOC-related publications with sub-topics from reviews [6,8,9], combination of the estimations of SOF and SOH [52-56], resistance or impedance-based calibration [57-60], mechanical stress-based calibration [49], pack SOC [19,29,61,62], OCV-based model [63-65], electrochemical models [66-72], to algorithm improvements [73-95]. 
(SPKF) (e.g. [83]), unscented KF (UKF) (e.g. [18,87]), particle filter (PF) (e.g. [92]), sliding mode observer (e.g. [72]), proportional-integral observer (e.g. [95]), Luenberger observer (e.g. [96]), and genetic or neutral network algorithms (e.g. [13,23-28,82]). The improvements of algorithms are mainly reflected in the following aspects:

(1) The common ECMs are capable of describing first or second order characteristics in a linear battery system. To deal with more complicated electrochemical processes in batteries, models with higher [97-99] or fractional orders [72,100,101] would be desired in the regression. To satisfy this need, the compatibility of algorithms for high-order linear systems becomes more critical, since it allows the regression to use more complex models to yield higher precision.

(2) The algorithms become more adaptive for nonlinear systems, from earlier ones capable of handling linear systems to those able to handle linearized systems, strongly nonlinear continuous systems and systems merely calculable at each single point. Most practical systems often come with some extent of nonlinearity.

(3) The algorithms become more capable of handling noisy matrices that exhibit certain types of randomness, from earlier ones that require zero-sum and zero-mean Gaussian noise to recent ones that can deal with noisy matrices with quantitative expressions of randomness. In general, in measurement systems certain types of noise are inevitable and ubiquitous. The randomness in the distribution of noise (i.e. system errors) should be verified "white" and non-biased in a well-calibrated system. However, the noise from environmental interference (random error) may exhibit a characteristic distribution that is "not white" (biased). Improving the tolerance to biased noise is an important tactic for 
better accuracy in the regression.

In addition, the most difficult task of reviewing algorithms is to tell exactly which algorithm is the most accurate and make a definitive recommendation. This kind of conclusion is strongly case-sensitive: it involves almost every element in the estimation process, including the precision of battery model parameters, battery operating conditions, and especially the detailed construction of each algorithm.

\subsection{Dependency on data acquisition and Ah-counting}

\subsubsection{Can SOC estimation method be self-adaptive?}

A self-adaptive method is appealing for SOC estimation since battery performance is strongly time-dependent along its aging process. In pursuing such a capability, adaptability is unfortunately limited by the nature of the characteristics of the battery system, e.g. the $V_{O C}=$ $f(\mathrm{SOC})$ relationship; and the fidelity of its model in expressing such a relationship. First of all, the characteristics of a battery system, such as $V_{O C}=f(\mathrm{SOC})$, must be characterized precisely and accurately. Thus, the quality of the data in representing such a relationship must be high. This is true for both $e-S O C$ and $t-S O C$.

Because limited resources on a practical platform, SOC estimation is often performed by a preset process and database. The pre-assigned data used in the SOC estimation could be divided into two types: those that can be derived in real time, on line during normal operation of the battery and those that can only be obtained in laboratory (referred to as 'lab data' hereinafter). If all the preset data needed for successful execution of SOC estimation are the first type, this method could be completely self-adaptive. Therefore, to understand if a 
method can afford an adaptive SOC estimation is to analyze the necessities of lab data. Taking a typical KF method for example, the preset data needed in the estimation process are model parameters such as resistance and capacitance values in the ECM and $V_{O C}=f(\mathrm{SOC})$ curve. Due to the influence of temperature or $\mathrm{SOH}$, these preset data could vary during battery operation; thus, from time to time they need to be recalibrated after a certain period.

A few comments are worth noting here:

(1) On battery model parameters (and taking ECM for example): resistance and capacitance values in the ECM can be determined by various types of tests such as constant current or dynamic discharging and charging, which implies that identification of ECM parameters can be accomplished without disturbing the normal operation of battery. Therefore, this type of parameters can be adjusted online to improve the accuracy of estimation method over a long duration (hence these parameters could be adaptive).

(2) On the $V_{O C}=f(\mathrm{SOC})$ and the reciprocal SOC $=f^{-1}\left(V_{O C}\right)$ curve: (i) to obtain a reliable curve requires high precision time-consuming processes, e.g. galvanostatic intermittent titration technique (GITT) or quasi-static tests in controlled laboratory conditions (such as charging/discharging with an extremely small rate as $\mathrm{C} / 25$ ). This type of quasi-static tests cannot be conducted during normal use of battery. When one suspects the curve could have been altered, new lab data verification and calibration are required to update the calculation routine. (ii) The curve is indispensable. In the Ah-counting method, the need of this curve is straightforward: the initial SOC is out of reach by the integration process and can only be determined by tests and proper calibration. Although in Section 3.1 we also indicated that in addition to OCV other variables such as the impedance and the 
surface stress can also be used for calibration, the calibration using $\mathrm{SOC}=f^{-1}\left(V_{O C}\right)$ is the most accurate and reliable, widely used in the literature. For estimation methods with model-assisted regression process, the $\mathrm{SOC}=f^{-1}\left(V_{O C}\right)$ curve is a necessity when deducing the SOC from the estimated OCV value by the battery model.

Here we summarize the conclusion in this section: SOC estimation method generally needs a known SOC $=f^{-1}\left(V_{O C}\right)$ curve. Such an OCV-SOC relationship is supposed independent from the battery size, geometry, or shape; since it is supposed to be defined by thermodynamics if determined properly. However, through aging, such a relationship could vary, as influenced by variations in the active material compositions or their crystal structure, morphological changes in the electrodes, or cell balance. Examples can be found in some literature reports (e.g. $[30,31,50,51,102])$. The challenge is such a variation in the $\mathrm{SOC}=f^{-1}\left(V_{O C}\right)$ curve is difficult to detect and update using data obtained in the operation. It can only be recalibrated in the lab and updated in the software of the control unit. The development of self-adaptive model remains premature (or, at least, not vigorously validated) currently in most cases.

\subsubsection{The necessity of Ah-counting in model-based SOC regression: the issue of a reversed problem}

In Section 3.2, we pointed out that the Ah-counting methods are frequently invoked in model-assisted SOC regressions. From the publication analysis mentioned earlier, almost all the 50 papers on model-assisted SOC regressions involved Ah-counting, except two $[71,82]$. Although the reason for not using Ah-counting was not explicitly explained in the two exceptions, their selections are surprisingly coincident. Theoretically speaking, a valid model 
should be able to determine SOC independently with a two-step regression (i.e. the first step is to estimate OCV mostly from the value of terminal voltage, and the second step is to calculate the SOC from the estimated $\mathrm{OCV}$ ). So, why these regression models need to engage Ah-counting?

Here, the general framework of battery modeling approach may offer some explanations. Regardless what kind of battery models (i.e. ECMs, electrochemical models, neural network-, genetic algorithm-, or other artificial intelligence-based models) presents, battery regression models basically describe the relationship between the inputs (e.g. battery voltage and current) and OCV (as the output for inferring SOC). In other words, although models differ by their components and mechanics that correlate the inputs and output, their basic functions are the same. For ECMs, the components are resistance, capacitance, Warburg element, etc. and the mechanics is the ECD and the Kirchhoff laws. For electrochemical models, those are partial differential equations depicting the conservation laws of active species transport and kinetic expressions of electrochemical reactions. Analogously, in neural network models, the number, content and weight of neurons and the network configuration are the essence. The need for Ah-counting is to have an anchor to provide a point of reference for these model frameworks to assure the precision. Here, we shall use a typical first-order ECM (Figure 2) as an example to illustrate the point: 


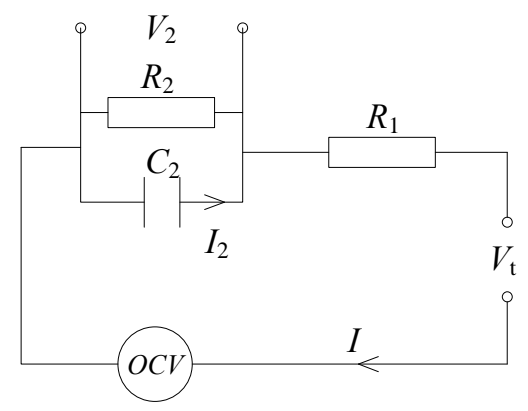

[Figure 2]

In this figure, OCV has a pre-determined relationship with SOC. $R_{1}, R_{2}$ and $C_{2}$ are circuit elements. $V_{2}$ is the divided voltage on the $R_{2} C_{2} . V_{\mathrm{t}}$ is the terminal voltage. $I$ is the current of the main loop and $I_{2}$ the current on the $C_{2}$ branch.

The differential equation of the circuit is:

$$
\begin{aligned}
& O C V=V_{t}-R_{1} I-V_{2}, \\
& \dot{V}_{2}=-\frac{1}{R_{2} C_{2}} V_{2}+\frac{I}{C_{2}} .
\end{aligned}
$$

Considering the concept of capacitance, $V_{2}$ also satisfies Eq. (13) and (13.1):

$$
\begin{aligned}
& V_{2}=Q / C_{2}, \\
& Q=\int I_{2} d t .
\end{aligned}
$$

where $Q$ is the capacity of $C_{2}$, and it's the integration of branch current $I_{2}$ over time.

Prior to the analysis of the three items on the right-hand side of the OCV formula in Eq. (12), we should introduce the concept of "frequency range" or "response time constant" to describe the response characteristics of other variables in Eq. (12) and (12.1) when the circuit on the main loop is excited with high-frequency alternative current signals. Some additional denotations should be explained here:

- High frequency parameters - when the current on the main loop $I$ is of high frequency, 
the branch current $I_{2}$ and terminal voltage $V_{\mathrm{t}}$ are both of high frequency.

- Low-frequency parameters - A one-time integral of the high-frequency parameters generates parameters with low frequency, such as $Q$ and $V_{2}$, given by Eq. (13) and (13.1), and SOC, expressed in Eq. (1). Since SOC and OCV have an algebraic correlation denoted by a rather smooth curve, the OCV is also assumed low frequency.

- Algebraic calculation rules between high and low frequency parameters - the algebraic sum of high and low frequency parameters is of high frequency. Since the main loop $I$ is of high frequency and the low-frequency $V_{2}$ gives the current on the $R_{2}$ branch low frequency), it explains why the branch current $I_{2}$ is of high frequency.

Thus, as deduced from Eq. (12), the three items on the right-hand side of OCV, $V_{\mathrm{t}}, R_{1} I$ and $V_{2}$, are of high, high and low frequency, respectively. Only when the difference between the former two items is of low frequency, the OCV result calculated by the formula in Eq. (12) would be of low frequency. Therefore, we can attain a low frequency SOC according to the above rules.

Whether or not the difference between $V_{\mathrm{t}}$ and $R_{1} I$ is of low frequency depends on the value of $R_{1}$. When the identification process of $R_{1}$ is assumed completely accurate, the high frequency part of the difference can be theoretically eliminated. But the deviation to this ideal situation often occurs in reality due to the reasons as follows:

(1) The identification of $R_{1}$ will inevitably have errors. This is because the identification accuracy is affected by various factors such as battery temperature and its fluctuations. In real working conditions, the temperature of the battery over the test process is neither identical temporally nor uniform spatially. Therefore, the identified $R_{1}$ could only achieve 
accuracy in the sense of averaging over a certain period of time.

(2) The high frequency parameters, $I$ and $V_{\mathrm{t}}$, could be slightly different in phase in actual measurements due to data acquisition system constraints, a phase difference in the magnitude of milliseconds could result in non-negligible high frequency difference between $V_{\mathrm{t}}$ and $R_{1} I$.

Therefore, deviating from theory, slight disturbance in real computation process could generate a high frequency estimated SOC (which is against its nature since SOC is the result of integral) when only using battery models in the regression. This problem explains the irreplaceable value of the Ah-counting, which filters the impact of high frequency current and provides the stability for SOC estimation. When Ah-counting and model-based regression are used in conjunction, as in the typical KF algorithm, the recursive structure ensures that the SOC prediction inherits certain stability from the Ah-counting process.

Furthermore, we should point out that, under the high frequency current perturbation to the main loop, the process of predicting $V_{\mathrm{t}}$ from a known OCV could reach a much higher accuracy. It is because this process basically deals with an algebraic calculation between high frequency $R_{1} I$ and low frequency OCV, $V_{2}$. Such a computation to yield a high frequency $V_{\mathrm{t}}$ could be easily achieved since the frequency of the result is not sensitive to calculation errors. Such a reverse calculation is the most commonly used in verifying the accuracy of the battery models.

In summary, if taken the calculation in the above paragraph as a forward process due to its common usage in validating battery models, its reversed process, to obtain a low frequency result by subtracting one high-frequency input from another, could be quite risky and have a 
greater probability of getting a result with high frequency oscillations. This explains the observation in Section 3.4.2 regarding the strong engagement of Ah-counting in most of the model-assisted SOC regression methods.

\section{MODELS}

Models used in the regression process have different flavors. As an example, most of the neutral network and genetic algorithm models emphasize empirical correlations in the models that consider battery as a "black box." In this section, we shall focus on physical models that emphasize the mechanics of battery behavior as regarded in "multi-physics-based models." The overarching task here is how to construct such models that are sufficiently accurate to assist SOC estimation.

\subsection{Multi-physics models and coupling principles}

When composing multi-physics models, one has to keep in mind that although SOC is a metric of expressing the degree of capacity utilization, to depict this aspect with sufficient accuracy and precision the coupling of the electrical properties with other properties of the battery is needed. In principle, three physical properties (electrical, thermal and mechanical) of a battery need to be considered in such coupling mechanism in the model development.

To provide a better perspective on how to address the coupled physical processes with proper degree of consideration, here we use the concept of "frequency range" or "response time constant" as one critical aspect in the coupling mechanics. Through analyzing the time constant of the (energy) transfer functions, expressed as the inputs and outputs of the battery system in Table 5, the mechanics of this coupling mechanism could be analyzed according to 
the frequency range of different physical properties.

\subsubsection{Response time constant of the electrical property}

The EIS technique is capable of pinpointing the time constant of the electrical property in a battery system. A result of an EIS measurement is exemplified in Figure 3 as expressed in a Nyquist plot. In the Nyquist plot, the characteristic of the response time of the electrical property of a battery, such as that in this example, covered a wide frequency range, from 1 $\mathrm{kHz} \sim 1 \mathrm{MHz}$, a range corresponds to the impedance of electron flows in the circuit, to $<1$ $\mathrm{mHz}$, which corresponds to the intercalation/de-intercalation of ions during DC discharging or charging processes that induces substantial SOC change.

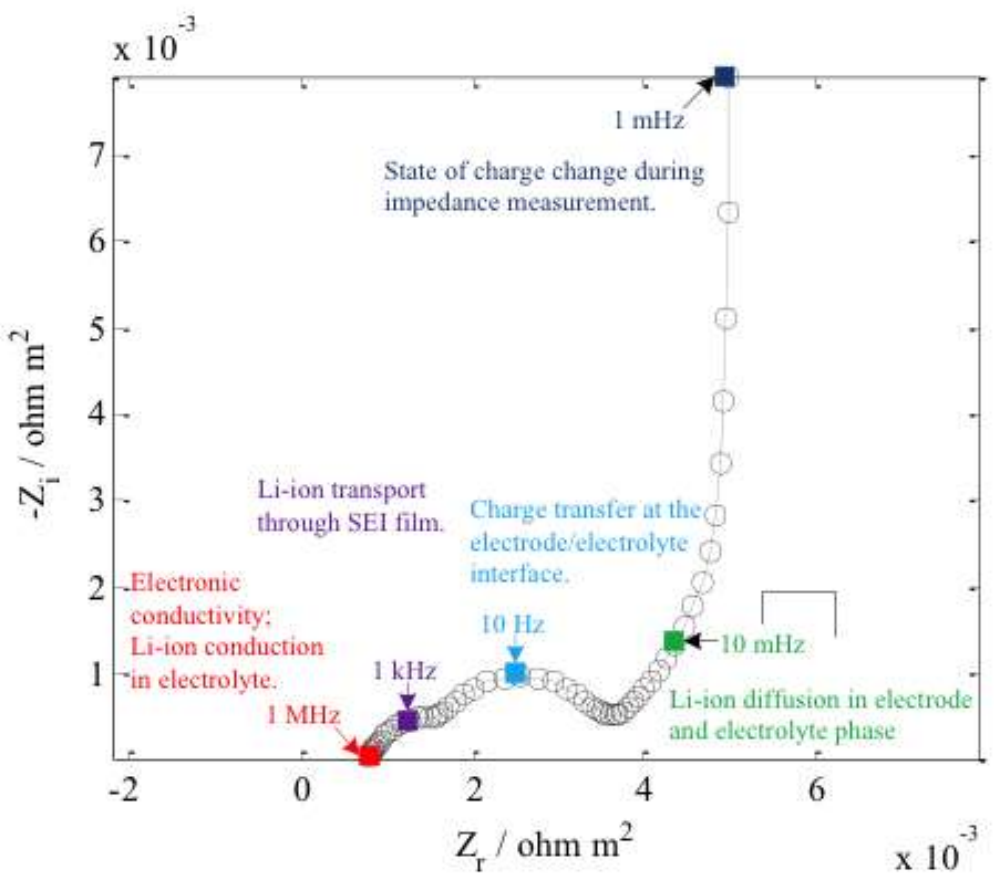

[Figure 3] 


\subsubsection{Response time constant of the thermal property}

The frequency range covered with regard to the thermal property is much narrower than that of electrical one, and this range usually lacks the high frequency region. To figure what range it covers, the following methods are introduced:

\subsubsection{A rule of thumb}

Thermal response time of a material is determined by the thermal conductivity of the material in the direction of the heat flux. Hence, the response time constant can be easily estimated by:

$$
\tau_{\text {thermal }}=R_{\text {thermal }} \cdot C_{\text {thermal }}=\frac{L}{\kappa} \cdot \rho C_{p} L
$$

where $\tau_{\text {thermal }}, R_{\text {thermal }}$, and $C_{\text {thermal }}$ are the time constant, thermal resistance and thermal capacitance between the heat source and the measurement point, respectively. $L$ is the distance between the two locations, $\kappa$ the thermal conductivity, $\rho$ the material density, and $C_{\mathrm{p}}$ the thermal capacitance of the material.

\subsubsection{Experimental method}

By placing embedded temperature sensors at different locations in a laminated cell, the thermal response time of the cell could be determined along the separation distances [103]. The procedure is to situate the battery in a step heating process and use the transfer function of the first-order inertia process to obtain the correlation between the temperatures measured in the environment and those inside the cell or on its surface. The experimental procedure and fitting process of such an experiment as an example is described in detail in Ref. [103]. Figure 4 exhibits the results of this example in which the thermal time constant of a cell subjected to a series of experiments with external heating was derived. Figure 4(a) and (b) 
show the time constants measured between the temperature transducer placed in the ambient environment and the one inside the cell or on the surface of the cell, which are on the order of $400 \sim 650 \mathrm{~s}$. The difference of the two yields the time constant for the heat conduction in the cell. The thermal time constant within the cell is on the order of $20 \sim 70 \mathrm{~s}$.

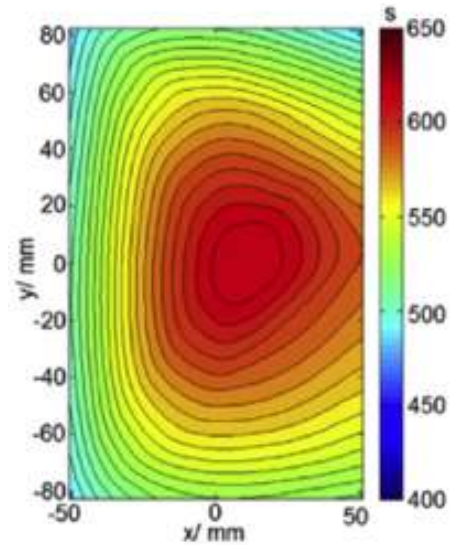

(a) Inside

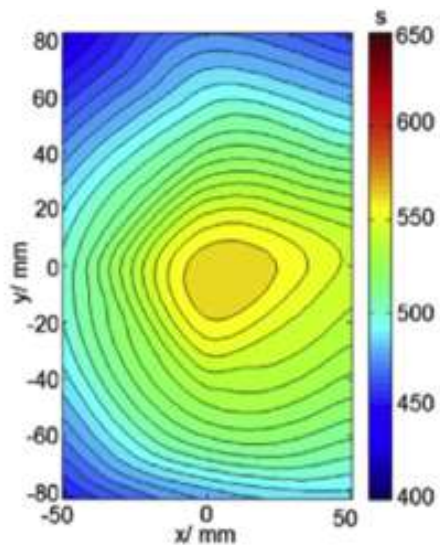

(b) Surface

[Figure 4]

This result is quite similar to the one calculated by Eq. (14), thus,

$$
\tau_{\text {thermal }}=\frac{L}{\kappa} \cdot \rho C_{p} L=\frac{\left(3.6 \times 10^{-3}\right)^{2}}{0.48} \times 2300 \times 1243=77 s,
$$

where the parameters of the cell geometry are listed in Table 6 and the thermal conductivity $\kappa$ and the thermal capacitance of the material $C_{\mathrm{p}}$ are both pre-determined by the optimization method developed in Ref. [104].

\subsubsection{Response time constant of the mechanical property}

As Table 5 shows, the output of mechanical property is more complex; therefore, its response time may have to be defined in more diverse ways. When the stress of a clamped battery or the volume of a battery in a free state is considered, these mechanical properties as outputs of 
the battery can be evaluated within the duration of one single charging or discharging regime, and the time constant is on the scale of several minutes. However, when the change of these outputs is concerned, the temporal scale shall actually reflect a slow aging process. Under non-abusive aging conditions, this time constant could be in months or years.

\subsubsection{The principle of multi-physics coupling}

The layout of the time constant span of each phenomenon of the three physical properties in a battery on a temporal coordinate is sketched in Figure 5. When selecting a battery model for SOC estimation under the principle of multi-physics coupling, we only need to couple those properties that work in the same frequency range according to the working condition and those on higher frequencies. From this perspective, the coupling could be treated in the following three aspects as follows:

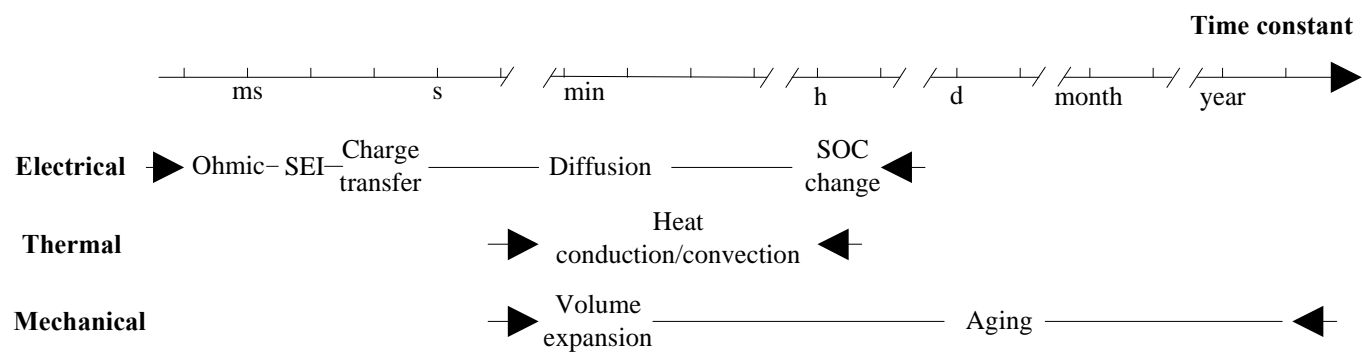

[Figure 5]

(1) When estimating the SOC fluctuations within a few seconds or less than one minute, it's quite likely that the temperature, stress and volume of the battery remain rather constant within this short interval. Thus, an electrical model without coupling with thermal or mechanical properties shall serve the purpose. 
(2) When estimating the SOC changes for intervals from a couple of minutes to a few hours, thermal effects should be considered through a thermal-electrical coupling model. If the system's volume changes and/or stress field variations are significant, the coupling should be extended to include mechanical properties.

(3) For studies on the cumulative changes of SOC in days or longer, such as self-discharge during storage or re-calibration of SOC estimation after a long time, the impact of battery aging should be fully considered in the battery model. Coupling of the three properties is a necessity to realize the precision of SOC estimation over a long period.

Based on the coupling principles stated above, we should now discuss the best practice to satisfy the criteria. This issue touches the essence of parameterization in the electrical (ECM or electrochemical), thermal and mechanical model coupling. Prior work in this area has been limited and likely incomplete. For example, electrical and thermal model coupling has been reported [105], but the results are still far from sufficient to elucidate the interactions between any two of the three basic properties of coupling. Any attempt to explain every possible coupling is an enormous task, probably outside the scope of this article. The schematic in Figure 6 just illustrates part of the important aspects in this triangular relationship of coupling.

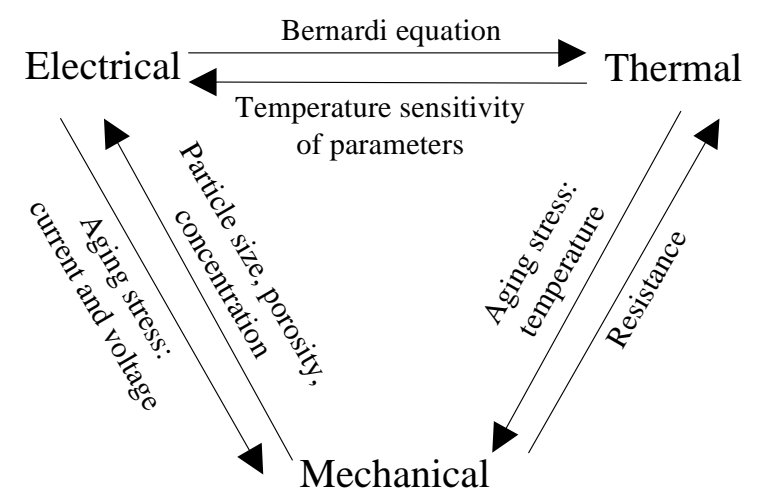


[Figure 6]

The coupling between electrical and thermal properties are the most commonly-used in battery modeling to predict SOC. In Sections 4.2 and 4.3, we shall discuss critical aspects in these two properties: i.e. the degree of simplification in electrical and electrochemical models and the dimension reduction of the thermal models, respectively.

\subsection{Electrical model: mechanistic or equivalent?}

In dealing with electrical responses of batteries under polarization, electrical models can be categorized generically in two types: electrochemical models and ECMs, according to the degree of simplification of the reaction mechanism. The electrochemical models are a family of 1-D or quasi-2-D models originated from Newman and his co-workers [106,107]. Newman's model is based on basic conservation laws, including mass transfer equations such as those depicted by Fick's laws and electrochemical principles to express the temporal and spatial relationships among the $\mathrm{Li}$ ion concentration distribution, the potential at the solid/electrolyte interface and the current density cross the surface of the active materials. The parameters of electrochemical models are dynamic coefficients describing the electrochemical reaction or mass transfer behaviors. Several refinements and extensions based on the original Newman model have been progressed continuously in the last few decades; for instance,

- Incorporation of the element of electric double layer at the interface to enhance accuracy of depicting the dynamic response of voltage to current steps.

- Denoting the edge effect of electrode plate by an extra surface-integral equation to allow 
two-dimensional simulations.

- Incorporating side reactions to model battery aging behavior, such as Li deposition on the negative electrode, including the threshold and side reaction intensity [108-110].

In contrast, ECMs are more comprehensive, especially for those who are familiar with circuit theory. In ECMs, the electrical elements such as resistance $(R)$, capacitance $(C)$ and Warburg impedance $(W)[99,111]$ are used in the model to describe the electrical behavior of a battery, where transfer functions are with current as the inputs and terminal voltage the output. Improvements of ECMs have grown through a number of stages over the past couple decades, resulting in a few better-known ones such as those under Rint, RC, Thevenin, Randle, PNGV, Warburg-included PNGV models. The objective among these variations remains the same: by placing $R, C$ and $W$ elements in different configurations, researchers aim to improve fidelity and precision of the model predictions according to the data they have in hand. It is difficult to tell if such distinctions truly reflect the physical reality of the electrochemical reactions involved in a proper temporal and/or spatial manner. For temporal resolutions, the goal is to seek understanding of possible physical processes incurred in the battery via the separation of time constants in the frequency scans, whereas for spatial resolutions it seeks identification of locations where these possible processes may occur.

Accordingly, it is perceived that an electrochemical model is more complicated than an ECM since the complexity of parameterization involved in the former is much higher than the latter. Table 7 provides a brief summary of the distinctions between the two approaches in modeling.

So far, to our knowledge, not a single paper has addressed all the parameterization issues in 
an electrochemical model with sufficient level of validation through design of experiments, let alone the uncertainty in accuracy. Therefore, it is fair to say that electrochemical models are useful in guiding semi-quantitative assessments of battery designs and their performance. Although attempts to use electrochemical models for power control-orientated functions have been pursued (e.g. in Ref. [112]), the practicality remains to be demonstrated. Nonetheless, such efforts may deliver merits and utilities that ECMs may not be able to offer, such as:

- The inherent ability to predict battery performance in the framework of forward models and computer designs with a variety of dynamic ranges of geometric parameters and test conditions to guide battery design and development.

- The ability of incorporating multi-reaction scenarios that include side reactions to address aging and degradation issues (e.g. in the study of lithium deposition [109].)

On the contrary, ECMs are convenient to derive empirical results from evaluations and data analyses. The reduction in computation resources makes ECMs a preferred choice for many engineering practices, including SOC estimations and for mobile, online, or embedded applications. In addition, the ECMs are more likely to achieve higher precision in practices due to its inherent merits in simpler parameter identification.

\subsection{Thermal model: lumped or distributed?}

The dimension selection of battery thermal model in the literature is highly diverse, from mass-point, zero-dimensional ones to one-, two-, or three-dimensional. Table 8 summarizes the selection of dimensions in various approaches in the literature.

The three-dimensional coordinates of batteries of three different structures in Table 8 are 
critical for the selection of the thermal model dimensionality, which should be based on the extent of unevenness in the temperature distributions (or thermal gradients) in a battery. In general, the stronger the non-uniformity in temperature distributions the battery exhibits in a scenario, the higher the need of increasing dimensionality in the thermal modeling. The following guidance might be useful in the model dimensional consideration:

(1) The larger the battery size, the more severe thermal non-uniformity the battery exhibits. It is common to assume a uniform temperature distribution to describe its overall thermal state of a battery. Such an assumption presents higher risks of losing fidelity and accuracy as the size of the battery increases. Thus, increasing dimensionality should be considered as the battery grows in size and complexity. At least, it is recommended to include the dimension of which the largest thermal gradient is expected such as the in-plane direction of a laminated cell [103] or the thickness of a thick prismatic cell.

(2) If the scales of a battery in different dimensions are distinct, non-uniformity in thermal energy distributions could exist. High dimensional thermal models should be considered in this case. Such a scenario is often the case for laminated cells, since the dimensional variability is high in these cells. For instance, the thickness of these cells is generally a few millimeters, but the width and length of the electrode plane could be up to tens of centimeters.

(3) Non-uniformity in thermal energy distributions is also frequently present in batteries with materials that have a strong dimensional dependence on thermal conductivity or electrode design. An example shown in Ref. [104] showed that for a laminated battery the thermal conductivity along the thickness could be $1 / 40$ of that in the in-plane direction. It should 
be noted that, in this example, although the thermal conductivity in the thickness direction is much smaller, the temperature uniformity in this direction is still better than that of the in-plane direction. This is because the temperature uniformity is an integral product of a few attributes, including the dimensional anisotropy in thermal conductivity, the position of the heat source, the flux of heat dissipation in different directions, and the disparity of the dimensional scales.

(4) For a battery system on a vehicle platform, the influences from the design and control of the thermal management system should make the thermal energy distribution even more complex. In this case, high-dimensional thermal models should be employed to reveal the temperature variations of cells at different locations, so adequate adjustments to the SOC algorithms could be placed in line with such variations.

In high-dimensional thermal models, identification of the anisotropic thermal parameters of a battery is the main challenge to improve thermal model fidelity and accuracy. Taking laminated cells as examples, the difficulty of parameter identification may include the following aspects:

- Due to the presence of electrolyte (mostly liquid) inside the battery, thermal parameters have to be measured in situ in order to reduce any discrepancies in model predictions. Methods performed on a stack of dry electrodes and separators often cannot achieve satisfactory precision [113-115].

- Typical laminated battery comprises of different materials on the through-plane direction, constituted by layers of electrodes and separators. To ease model computation, it requires an equivalent thermal conductivity in this direction. To measure this quantity, instrument 
error could be considerable [116,117,118].

- Thermal property of the Al-plastic film packaging for laminated pouch cells is different from the cell interior core, requiring to be considered separately.

- Layers of electrodes and separators in stacked or wound configurations could generate additional heat resistance between layers (partly due to the variation in the strength of mechanical contact). This variation is difficult to estimate; implying that, without careful validation, using an apparent thermal conductivity based on the collective conductivity of individual materials along the through plane in the model calculation may not be accurate anymore.

Conducting in situ identification of anisotropic thermal conductivities could be extremely challenging and it is beyond the scope of this article. However, the authors have explored a joint-optimization method based on the experimental thermal response of the battery during external heating, and the simulation results [104] seemed to suggest that satisfactory precision could be achieved for the determination of anisotropic thermal parameters of laminated cells.

\subsection{Model validation}

Validating models could be as challenging as establishing them. Most of the time, we are unable to perform validation correctly and sufficiently due to possible errors that undermine the validation. These errors could originate from various sources. In conducting experiments, the bias in collecting data from transducers, protocols, or procedures could introduce errors systematically, which are difficult to manage already inherently. Barriers in validation could 
also come from the lack of sufficient temporal or spatial resolutions, since validation is often carried out at a single location or at a specific interval, easily leading to biased result when the system exhibits non-uniformity issues.

Temperature is the most commonly used variable to validate a thermal-electrical coupled model of batteries. Table 9 summarizes two approaches of measuring temperature and the potential error origins, as well as possible solutions in each approach.

Even if the transducers used are accurate and the information from these transducers has been correctly adjusted and interpreted, the model validation may be still far from sufficient when temporal or spatial resolutions have not been fully investigated. Table 10 summarizes several solutions to alleviate the insufficiency of validation, both temporally and spatially.

\section{ACCURACY}

\subsection{Demands on accuracy}

Since the 1970s, 3C products have initiated the large-scale use of AA-, 18650-size, and flat wound lithium-ion batteries. The remaining capacity of the battery in typical $3 \mathrm{C}$ products, such as Walkman's, cell phones, and cameras, is displayed in real time on its user interface to alert the users when the battery may run out of power so they can timely replace or recharge the battery. Even though interruptions in the operation of these electronic products by the battery create inconvenience and nuisance, the scenario hardly causes emergency or safety concern to the users. Thus, the users and the product developers have tolerated inaccuracy in SOC estimations with patience. Since 2000s, emerging telecommunication and information technology business needs reliable power sources that can provide mobility and portability 
with battery-powered devices. Such a demand has shaped more stringent requirements for accurate SOC estimation, for the following reasons:

(1) Demands in cost-effective high energy and high power operation have driven the change in the battery chemistry from primary to secondary and from nickel-metal hydride to lithium-ion. The lithium-ion battery designs are prone to draw concerns on safety and reliability due to their inability to tolerate abuses. For instance, lithium-ion batteries are sensitive to overcharge and overdischarge. Overestimated or underestimated SOC would easily lead to overcharge or overdischarge of the battery. Such abuses could result in irreversible capacity loss or even safety incidents [139].

(2) Demands in environmental-friendly renewable energy applications, such as in EVs or grid energy storage systems where much higher quantities and larger sizes of battery cells in the system are required, are penetrating the consumer market and replacing $3 \mathrm{C}$ products as a more dominant share of battery supply chain. This demand-and-supply situation has also changed the playing field of battery technology and its development in many aspects. For example, for the EV applications, reducing volume and weight for the battery system are all critical design criteria, which leave little safety margin for the battery system to withstand abuses or address reliability issues by the conventional practice of mitigating abuses by overdesign the tolerance in performance envelope. All battery-manufacturing processes now have to be precise and accurate to meet every design requirements. The stringent requirement may well include the SOC estimation, as inaccurate estimates can lead to a malfunctioning vehicle and fatal consequence. Furthermore, the uncertainty for the SOC estimation shall result in more conservative battery usage, lower utility and 
mobility as well.

These demands drive the pursuit for more accurate SOC estimations in the research. In the literature reports, improvements on accuracy have been claimed from early $8 \%, 5 \%$, to $2 \%$ or even $1 \%$ in recent years [19]. To ensure practicality and economy of the SOC estimation methods and provide a trustworthy guidance for developing usable applications, the following protocols on how to determine a reasonable accuracy are suggested:

(1) For BEVs, the tolerance or requirement for SOC accuracy could be set at about $5 \%$. The proposed protocol is based on the allowance of usable capacity. At the lower end of the capacity range, the convention used by the OEMs usually sets the cutoff at more than $10 \%$ SOC to account for the possible error of 5\% inaccuracy, which leaves the users with the very last 5\% capacity for finding emergency charging or other solutions. For a typical EV equipped with a $20 \mathrm{kWh}$ pack, $5 \%$ capacity should allow additional $3 \sim 5 \mathrm{~km}$ of driving. To alleviate overcharging at the higher end of the capacity range, the manufacturers usually take a conservative stance, e.g. $0.1-0.2 \mathrm{~V}$ below the safety limit. The margin of $0.1-0.2 \mathrm{~V}$ could enable the battery to accommodate additional 5\% - 10\% capacity, if necessary. In some cases, batteries could withstand $120 \%$ SOC overcharge without obvious capacity degradation [139]. If so, a battery may survive a 5\% SOC underestimation, since an equivalent amount of overcharging might not lead to severe consequences.

(2) For hybrid EVs, a typical demand for accuracy could be the same as 5\%, but this protocol comes out of a different reason. This margin arises from the need of transient control in the vehicle powertrain. Considering the hybrid system comprising the battery and the internal combustion engine (ICE), the response time to power switch of the battery-motor 
system is several magnitudes shorter than that of ICE system [140] (say, ms vs. s). To handle such a disparity in the temporal domain to warrant a smooth and accurate output in the powertrain operation, a typical power control unit usually employs a conventional strategy that fixes the power output of the ICE at an appropriate level and adjusts the power output of the battery spontaneously. Here the maximum power capability of the battery is crucial since the matching power output of the ICE should be determined in advance accordingly, and the key parameters used in the determination of the battery power capability is strongly related to the SOC [140]. In general, at low SOCs, the DC resistance (DCR) of a battery increases noticeably [34] and the maximum power output rapidly reduces. Figure 7 shows an example in which a battery in a hybrid EV is supposed to operate in a power assist mode $<4>$. If the battery operates in the middle SOC range, say $40 \% \sim 60 \%$, a variation of $5 \%$ SOC could induce a $<5 \%$ deviation in the battery power capability. As the SOC decreases, the battery power capability also decreases rapidly and becomes more sensitive to the errors in the SOC estimation. Since the power control of hybrid EVs generally requires an error $<5 \%$, the accuracy of the SOC estimation should be set as $5 \%$ if the DCR curve in Figure 7 is adopted. It should be noted that since the DCR curve is different for types of batteries and the SOC range in use on hybrid EVs could be different too, this $5 \%$ standard is subject to change.

<4> Different from BEVs, hybrid EVs only use a narrow SOC range of its battery. The set point for the power assist mode is usually near the center of the SOC range and has a span of around $20 \%$. This set point may drift as the battery ages. For plug-in hybrid EV (PHEV), the center point shall vary since this type of EV has a preceding pure battery drive mode before the powertrain goes hybrid. Generally, batteries of PHEV have a wider SOC operation range than HEV. 


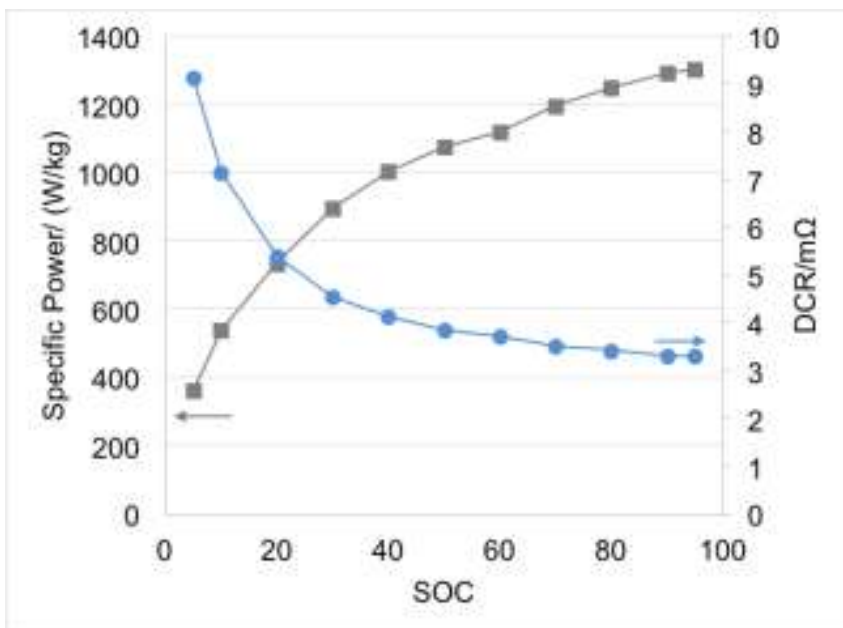

[Figure 7]

It is worth noting that the hybrid EVs are equipped with smaller batteries, typically about 1 2 $\mathrm{kWh}$, much smaller than those of BEVs typically in the range of $15 \sim 25 \mathrm{kWh}$. Under this circumstance, even at lower SOCs such as $20 \%$, the power capability of BEV batteries often could meet the demands in the powertrain. Thus, no additional protocol is needed from the perspective of power control when deciding an appropriate level of SOC accuracy for BEVs.

In summary, 3C products and EVs have specific and unique requirements on SOC accuracy, and those for EVs are stricter. For EVs, pursuing the last $1 \%$ of SOC accuracy is not only exhausting and costly but also unnecessary at most scenarios. In general, a $5 \%$ of SOC accuracy could meet the demand of most existing types of battery-powered products. The real challenge is not the accuracy of $e-S O C$ against the experimental data but $t-S O C$, so system engineers would not catch surprises that their supposedly precise and accurate $e$-SOC does not match up with reality (i.e. $t-S O C$ ). 


\subsection{Going statistical}

It is important to recognize that many factors affect the accuracy of SOC estimation. Sound statistical practices are critical to yield the needed accuracy. The following factors could contribute to variability of data that are used in the derivation of models and methods for an accurate SOC estimation:

\subsubsection{Measurement noise}

The interference from experimental measurements exists in every step of SOC estimation. Taking KF method for example, such interference manifests in the state-space model when acquiring terminal voltage, OCV, current, and temperature, where uncertainties in readings from sensory transducers, recording devices, possible biases in the test protocols and procedures could be introduced into parameter identification for the battery model. Such interference also appears in the observation space where errors in the current and the time keeping of the Ah-counting, so measurement noise would generate an error bar on the estimated results. In other words, a confidence interval for each measurement would be carried over into precision and accuracy of the prediction. The error bars or confidence intervals are associated with the measurement noise matrix.

\subsubsection{Spatial non-uniformity inside large-format single cells}

Spatial non-uniformity exists in large-format single cells. For conventional size batteries, e.g. AA, 18650 and other small ones, the temperature inside and outside a single cell is often considered rather uniform. For example, a case study reported that the temperature variations 
among different locations on the surface of an 18650 cell should be no more than $2^{\circ} \mathrm{C}$ [141]. However, for large-format batteries with a capacity more than $10 \mathrm{Ah}$, the temperature could be significantly different. For example, a case study with a 25 Ah laminated cell discharged in an environment chamber with forced ventilation showed that the temperature variations among different locations on the surface could be as high as $8^{\circ} \mathrm{C}$ at a mild discharge rate such as $1.5 \mathrm{C}$ [103]. Even the cell has a thin structure only $7 \mathrm{~mm}$ thick, one point on the surface and another internal point just $3.5 \mathrm{~mm}$ below the surface could have a $2^{\circ} \mathrm{C}$ difference [103].

The spatial non-uniformity of temperature in a cell shall lead to a difficulty of describing the state of the cell using a single-point data. Since the key parameters of the battery model, such as resistance, current, or capacity, are all closely related to the temperature distributions, noticeable spatial non-uniformity may render a model not trustworthy under a uniform assumption. Possible solutions are suggested as follows:

(1) Lumped approach: to use several temperature sensors placed at different locations of a large-format cell and derive a synthesized temperature based on the mean, median or a spatially weighted average as a representative temperature for the cell in the model. The lumped approach may reduce the error of a single-point temperature input, but it cannot completely compensate the impact of spatial non-uniformity on the accuracy of the model predictions.

(2) High-fidelity model coupling approach: incorporate the temperature non-uniformity and its interdependent phenomena by coupling the thermal aspects into the electrical model. This coupled model should adopt the true geometry, not necessarily three-dimensional but at least dimensional dependence on the temperature gradients. In multi-physics models it 
is a mutual coupling, where the electrical (i.e. potential/voltage and current) and thermal variables (i.e. temperature) are interactively and reciprocally correlated. On one hand, the heat generation rate is a function of overpotential, as expressed in the model by Bernardi et al. [142], whereas the overpotential is predicted by the ECM or electrochemical model $[105,107,143]$. On the other, the resistance in the ECM, the diffusion and reaction coefficients in the electrochemical models are all temperature-dependent. The multi-physics models afford us a complete description of incorporating spatial non-uniformities in single cells and clarifying the interactions between the thermal and electrical aspects.

However, to establish such three-dimensional multi-physics models with high fidelity is not easy in reality and the difficulties may root in all aspects of modeling issues, such as the coupling methods, the geometric construction and the accurate determination of electrical, electrochemical and thermal parameters. Introduction to these difficulties and the know-hows to construct an accurate model have been discussed in details in Section 4.

\subsubsection{Cell-to-cell variation}

Cell-to-cell variation is a great concern in addressing the accuracy issues of SOC estimation. To express cell-to-cell variation, the common practice is to reply on statistical analysis (by a standard deviation from rated capacity or resistance for a specific cell model). Utilizing quality control and inspection at the manufacturing site is helpful to yield quality cells. However, the reliability of such control and assessment is hardly discussed in the literature. A study by Dubarry et al. [144] shed some light on the origins and identifications of cell-to-cell 
variation. A worthy note from this study is that battery performance metrics are a complex composition of thermodynamic and kinetic effects, of which each factor has a probability distribution by itself. As such, the statistical analysis on the metrics and their confidence level distributions are not a reliable characterization, since the sub-level distributions of its constituents might change over manufacturing processes and periods of storage time. This underlying effect has a significant impact on the precision and accuracy of the SOC estimation, which is difficult to characterize, since it is time-, origin-, and environment-dependent (or, in a more general term, path-dependent).

Figure 8 is an example to illustrate the points. Figure 8 (a) shows the distribution of the DC resistance (DCR) in a batch of 100 commercial cells [144]. This distribution may appear as a natural Gaussian one. However, DCR has profound impacts on charging process as exhibited in Figure 8(b), where the end-of-charge (EOC) trickle charge currents at the cutoff and the rest cell voltages (RCVs) over a long rest period that represent the final SOCs are shown to have a density map that does not retain the same DCR distribution among the same 100 cells. The subsequent discharge regimes at (c) $\mathrm{C} / 2$ and (d) $\mathrm{C} / 5$, respectively, show a more perplexing nature in the capacity distribution profiles, since they do not resemble each other, although the only difference is supposed to be just the discharge rate. This example illustrated a unique aspect of statistical analysis for battery performance: as we commonly believed that DCR has a strong influence on capacity, such an influence is not well validated as this example showed. It is arguably profound to realize that the capacity distribution of these cells does not follow a one-to-one correlation with DCR. In other words, the distributions of capacity and DCR bear no kin to each other, so any statistical correlation between capacity 
and DCR may not bear any meaningful relationship.
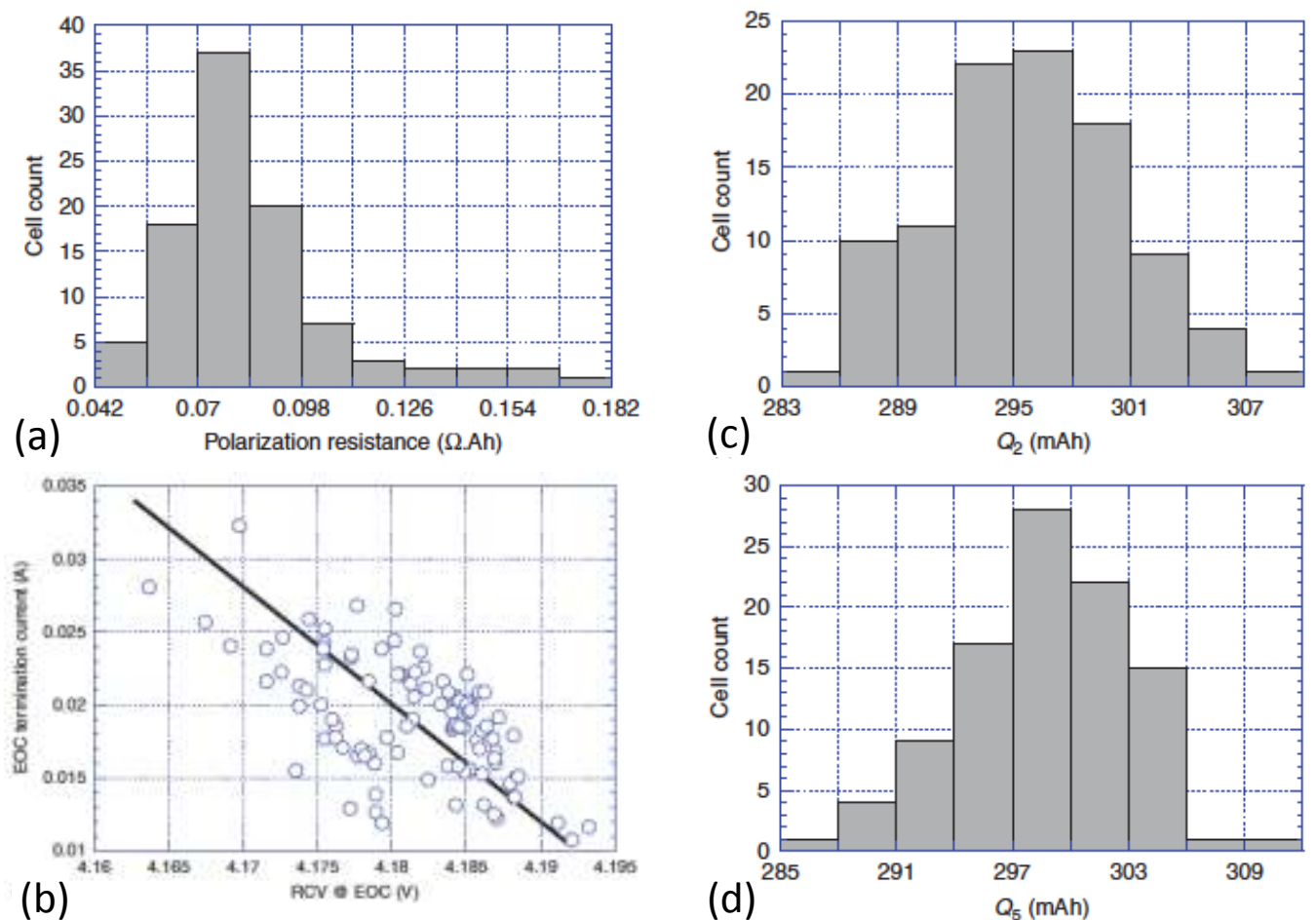

[Figure 8]

\subsubsection{Stacking cells in a battery pack}

Battery packs come with multiple-cell configurations and present additional challenges for SOC estimation. As these cells are connected in serial and/or parallel in the pack's wiring topology, several issues arise with SOC determination. An immediate consequence is the cell balance in the presence of the pack's wiring topology. The way a cell is connected in the pack with its neighboring cells shall create a unique causality in the cell's reaction to polarization. The capacity released from the pack is a collective result of each individual cells, implying a composition of divergent SOCs among the cells. How can we describe such an assembly for a proper SOC estimation of the pack? The following understanding could be used to derive a 
proper approach to determine the SOC for the pack.

1) Cells in series connections as in a string should have the same current passing through, thus releasing the same amount of charge. Due to cell-to-cell variation, the same amount of charge in each cell may not translate into an equivalent range of SOC variations engaged in the discharge, nor the same voltage drop in each cell.

2) Cells or strings in parallel connections shall have the same voltage across the terminals, thus spontaneous internal balance shall occur if their initial potentials are not the same. Those with higher potentials shall charge the lower ones until all reach the same potential. Such balance could be affected by other factors that lead to cell-to-cell variation besides potential. For instance, the cell internal resistance could be slightly different from one to another, which affect the current distribution in the branches among the cells or strings in parallel. Thus, the redistribution of the capacity due to this internal balance shall make the SOC estimation difficult not only for individual cells and strings but also for the pack.

3) Additional factors from the environment and harnessing infrastructure also contribute to the uncertainty of SOC estimation in the pack. For example, connectors of various shapes, sizes, and materials are used in the battery pack assembly. These bolts and nuts, welding in the structure, cables, and bus bars add to the resistance and impedance of the system. These connectors divert current, divide voltage, generate heat and dissipate energy, thus adding more unknowns to the SOC estimation by changing energy efficiency, diverting charge balance, and creating thermal gradients over non-uniformity.

4) Non-uniform temperature distributions in a pack seem inevitable, since the rate of heat generation and dissipation are often location- and direction-dependent. To ease the burden 
of on-board thermal management in a battery pack, the macro temperature variation over the pack should be controlled within a range, which has been optimistically set as $2 \sim 5^{\circ} \mathrm{C}$ by some EV manufacturers. In practice, managing temperature variations could be more challenging than controlling the maximum temperature since it involves the balancing of heat at multiple locations rather than just enhancing the ventilation at the hot spots. The temperature variations inside the battery pack could trigger the redistribution of current, affecting the charge balance, thus SOC estimations of individual cells in the pack or the SOC of the entire assembly.

5) The control strategy and function of battery management system (BMS) in a battery pack could also impose unintended consequential effects on the charge and discharge behavior of individual cells and the pack itself. Although the BMS should protect the battery pack from abuses that could result in severe adverse effects or accidents via monitoring and controlling the battery charge and discharge behavior, inappropriate control of the BMS, including inability to adapt for aging and degradation and improper balancing of cells in the pack, could also increase the uncertainty in the SOC estimations of individual cells and the pack.

Even though these effects might continue to interfere the goal of establishing a reliable SOC estimation, once we have better understanding and appreciation of the intricate coupling of electrical, thermal, and mechanical interactions among different elements in a battery pack we shall continue to improve the ability to deriving precise and accurate SOCs for cells and packs from basic principles and through careful validation. More recent work $[29,145,146]$ has pointed to this possibility with better technical understanding and strategy. An example is 
shown in Ref. [145] in which a battery pack with three cells in series is used for illustrating the method of determining SOC for the pack based on SOCs of the individual cells. Each of three cells suffered different degrees of capacity loss due to loss of lithium inventory (LLI), and such variations need to be taken care of in the derivation of proper correspondence of the SOC ranges used in the cells in the discharge regime in order to project the scenario for the pack. In this case, the SOC scale of each cell has to be translated into a coherent one in order to derive the one for the pack. Detail description of this scaling and calculation should be referenced to Ref. [145].

A battery pack configured with multiple single cells is an assembly of cells in isolation and interaction. On one hand, all cells are physically separated by packaging, implying the SOC of each cell may not necessarily resemble to each other; on the other, the electrical and thermal interactions among them never fully stop: current is redistributed, heat flux flows and efficiency adjusted. These interactions all contribute to the uncertainty in the SOC estimation of individual cells and the battery pack.

Finally in Section 5.2, it should be noted that, when discussing the error band of SOC estimation results, one should try to identify those error sources with deterministic features in advance, such as the effect of OCV hysteresis, to minimize the estimation error as much as possible and reduce any further work on error analysis.

\section{CONCLUSIONS}

It has been a long pursuit to achieve better SOC estimations of batteries over the past few decades, and a brief summary of the status of progress as presented in this work only tips off 
the first step to understand issues and challenges before we put a stake to the right directions. Here, as we indicated, a number of issues need further investigations to lay the foundation for a reliable SOC determination. A few key points are summarized here:

(1) Can we get rid of Ah-counting if we had sufficiently accurate battery models to correlate measurable variables (such as terminal voltage and current) with OCV? By analyzing the frequency range of each variable in a typical battery ECM, it is found that the estimated SOC may exhibit the characteristics of high frequency oscillation due to the inaccuracy of reversed algebraic calculation across frequencies. Incorporating Ah-counting in the model-assisted regression could help the SOC estimation converging due to the inherited stability from the integral process

(2) Constructing high-fidelity multi-physics models to perform SOC estimations accurately with the following suggestions:

a) Carefully taking into account the coupling among electrical, thermal and mechanical properties in the frequency range that is relevant to the working conditions.

b) Selecting proper modeling tools that can produce the needed utility. In most cases, the ECMs might be more appropriate than the electrochemical models mainly because of the likelihood of better precision in practice due to inherently less difficulties in parameter identification.

c) Considering higher-dimensions thermal model to accommodate uneven distributions of thermal gradients in the battery. At least, dimensionality along the largest thermal gradient should be considered. 
d) Validating the models should be carried out carefully to account for not only system errors from instrumentation and environment but also possible biases from protocols and sensor placement.

(3) The uncertainties in the SOC estimation could be attributed to several sources, including the measurement noise, spatial non-uniformity in a large cell, cell-to-cell variation, and configurational interference among cells in a battery pack. Therefore, it is intricate to apply statistical analyses with careful dimensional and temporal validation to establish confidence intervals based on physical understanding of the battery system and its characteristics, not merely empirical. Instead of being deterministic, the SOC estimation should enforce statistical results.

This article has gone beyond review to provide commentary aiming at helping stakeholders in this field making better decisions for their endeavors. We hope it will serve this purpose and help to advance the field. 


\section{Acknowledgments}

This manuscript has been authored by Battelle Energy Alliance, LLC under Contract No. DE-AC07-05ID14517 with the U.S. Department of Energy. The United States Government retains and the publisher, by accepting the article for publication, acknowledges that the United States Government retains a nonexclusive, paid-up, irrevocable, world-wide license to publish or reproduce the published form of this manuscript, or allow others to do so, for United States Government purposes. BYL would like to acknowledge that the statements and opinions in this article are solely those of the author. Nothing in this article represents the work, views or positions of the Idaho National Laboratory, the Department of Energy or any of their customers. BYL would also like to thank the travel support by the grants (KF14041 and KF16052) from the Foundation of State Key Laboratory of Automotive Safety and Energy at Tsinghua University, Beijing, China, to facilitate this collaboration. This work is supported by the National Natural Science Foundation of China under the grant number of 51577104 and 51377097. 


\section{REFERENCES}

1. M. Lurie, H.N. Seiger, R.C. Shair. State of charge methods for nickel-cadmium batteries. Proceedings of the 17th Annual Power Sources Conference (1963) 110-114.

2. C.W. Lillehei, A.B. Cruz, I. Johnsrude, R.D. Seller. A new method of assessing the state of charge of implanted cardiac pacemaker batteries. The American Journal of Cardiology 16 (1965) 717-721.

3. N. Latner. State of charge measurement of nickel-Cadmium batteries. Review of Scientific Instruments 40 (1969) 364; doi:10.1063/1.1683942.

4. F. Huet. A review of impedance measurements for determination of the state-of-charge or state-of-health of secondary batteries. Journal of Power Sources 70 (1998) 59-69.

5. S. Rodrigues, N. Munichandraiah, A.K. Shukla. A review of state-of-charge indication of batteries by means of ac impedance measurements. Journal of Power Sources 87 (2000) 12-20.

6. Z. Zou, J. Xu, C. Mi, B. Cao, Z. Chen. Evaluation of model based state of charge estimation methods for lithium-ion batteries. Energies 7 (2014) 5065-5082.

7. W. Waag, C. Fleischer, D.U. Sauer. Critical review of the methods for monitoring of lithium-ion batteries in electric and hybrid vehicles. Journal of Power Sources 258 (2014) 321-339.

8. J. Kalawoun, K. Biletska, F. Suard, M. Montaru. From a novel classification of the battery state of charge estimators toward a conception of an ideal one. Journal of Power Sources 279 (2015) 694-706.

9. A. Farmann, W. Waag, A. Marongiu, D.U. Sauer. Critical review of on-board capacity 
estimation techniques for lithium-ion batteries in electric and hybrid electric vehicles. Journal of Power Sources 281 (2015) 114-130.

10. L. Lu, X. Han, J. Li, J. Hua, M. Ouyang. A review on the key issues for lithium-ion battery management in electric vehicles. Journal of Power Sources 226 (2013) 272-288.

11. X. Wu, L. Mi, W. Tan, J.L. Qin, M.N. Zhao. State of charge (SOC) estimation of Ni-MH battery based on least square support vector machines. Advanced Materials Research 211 (2011) 1204-1209.

12. X. Hu, F. Sun, Y. Zou, H. Peng. Online estimation of an electric vehicle lithium-ion battery using recursive least squares with forgetting. Proc. of 2011 American Control Conference (ACC 2011), San Francisco, California, USA, June 29 -July 1, 2011, IEEE, 2011, pp. 935-940.

13. G. Li, Y. Xie, G. Li, N. Wei. Estimation model of battery SOC based on least square support vector machine optimized by genetic algorithm. Chinese Journal of Power Sources 9 (2012) 024.

14. H. Zhu, L. Gao, C. Qian. SOC estimation of power battery for electric car based on method of fuzzy least square support vector machine. Chinese Journal of Power Sources 5 (2013) 046.

15. G.L. Plett. Extended Kalman filtering for battery management systems of LiPB-based HEV battery packs: Part 1. Background. Journal of Power Sources 134 (2004) 252-261.

16. G.L. Plett. Extended Kalman filtering for battery management systems of LiPB-based 
HEV battery packs: Part 2. Modeling and identification. Journal of Power Sources 134 (2004) 262-276.

17. G.L. Plett. Extended Kalman filtering for battery management systems of LiPB-based HEV battery packs: Part 3. State and parameter estimation. Journal of Power Sources 134 (2004) 277-292.

18. F. Sun, X. Hu, Y. Zou, S. Li. Adaptive unscented Kalman filtering for state of charge estimation of a lithium-ion battery for electric vehicles. Energy 36 (2011) 3531-3540.

19. S. Sepasi, R. Ghorbani, B.Y. Liaw. Improved extended Kalman filter for state of charge estimation of battery pack. J. Power Sources 255 (2014) 368-376.

20. S. Sepasi, R. Ghorbani, B.Y. Liaw. Inline state of health estimation of lithium-ion batteries using state of charge calculation. J. Power Sources 299 (2015) 246-254.

21. A. Urbina, T.L. Paez, R.G. Jungst, B.Y. Liaw. Inductive modeling of lithium-ion cells. Journal of Power Sources 110 (2002) 430-436.

22. R.G. Jungst, G. Nagasubramanian, H.L. Case, B.Y. Liaw, A. Urbina, T.L. Paez, D.H. Doughty. Accelerated calendar and pulse life analysis of lithium-ion cells. Journal of Power Sources 119-121 (2003) 870-873.

23. I. Li, W.Y. Wang, S.F. Su, Y.S. Lee. A merged fuzzy neural network and its applications in battery state-of-charge estimation. IEEE Transactions on Energy Conversion 22 (2007) 697-708.

24. W.X. Shen. State of available capacity estimation for lead-acid batteries in electric vehicles using neural network. Energy Conversion and Management 48 (2007) 433-442. 
25. L. Xu, J. Wang, Q. Chen. Kalman filtering state of charge estimation for battery management system based on a stochastic fuzzy neural network battery model. Energy Conversion and Management 53 (2012) 33-39.

26. B. Cheng, Z. Bai, B. Cao. State of charge estimation based on evolutionary neural network. Energy Conversion and Management 49 (2008) 2788-2794.

27. S. Luo, X. Ma, Y. Lu. An advanced non-dominated sorting genetic algorithm based SOC hardware/software partitioning. Acta Electronica Sinica 11 (2009) 042.

28. T.O. Ting, K.L. Man, E.G. Lim, M. Leach. Tuning of Kalman filter parameters via genetic algorithm for state-of-charge estimation in battery management system. The Scientific World Journal (2014) Article ID 176052.

29. C. Truchot, M. Dubarry, B.Y. Liaw. State-of-charge estimation and uncertainty for lithium-ion battery strings. Applied Energy 119 (2014) 218-227.

30. M. Dubarry, V. Svoboda, R. Hwu, B.Y. Liaw. Incremental capacity analysis and close-to-equilibrium OCV measurements to quantify capacity fade in commercial rechargeable lithium batteries. Electrochemical and Solid-State Letters 9 (2006) A454-A457.

31. M. Dubarry, V. Svoboda, R. Hwu, B.Y. Liaw. Capacity loss in rechargeable lithium cells during cycle life testing: The importance of determining state-of-charge. Journal of Power Sources 174 (2007) 1121-1125.

32. W. Weppner, R.A. Huggins. Determination of the kinetic parameters of mixed-conducting electrodes and application to the system $\mathrm{Li}_{3} \mathrm{Sb}$. Journal of the Electrochemical Society 124 (1977) 1569-1578. 
33. USABC Electric Vehicle Battery Test Procedures Manual. Appendix F: Glossary of battery and battery testing terminology for the USABC battery test procedures, USABC, 2009. p. 1-10.

34. J. Zhang, J. Huang, Z. Li, B. Wu, Z. Nie, Y. Sun, F. An, N. Wu. Comparison and validation of methods for estimating heat generation rate of large-format lithium-ion batteries. Journal of Thermal Analysis and Calorimetry 117 (2014) 447-461.

35. Z. Li. A characteristic research on $\mathrm{LiFePO}_{4}$ batteries for pure electric vehicles. Dissertation, Tsinghua University, Beijing, China, July 2011.

36. W. Dreyer, J. Jamnik, C. Guhlke, R. Huth, J. Moškon, M. Gaberšček. The thermodynamic origin of hysteresis in insertion batteries. Nature Materials 9 (2010) 448-453.

37. M.A. Roscher, D.U. Sauer. Dynamic electric behavior and open-circuit-voltage modeling of $\mathrm{LiFePO}_{4}$-based lithium ion secondary batteries. Journal of Power Sources 196 (2011) 331-336.

38. T. Zheng, J.R. Dahn. Hysteresis observed in quasi open-circuit voltage measurements of lithium insertion in hydrogen-containing carbons. Journal of Power Sources 68 (1997) 201-203.

39. J.R. Croy, K.G. Gallagher, M. Balasubramanian, Z. Chen, Y. Ren, D. Kim, S. Kang, D.W. Dees, and M.M. Thackeray. Examining hysteresis in composite $\mathrm{x} \mathrm{Li}_{2} \mathrm{MnO}_{3} \cdot(1-\mathrm{x})$ $\mathrm{LiMnO}_{2}$ cathode structures. The Journal of Physical Chemistry C 117 (2013) $6525-6536$.

40. R. Khatib, A.-L. Dalverny, M. Saubanère, M. Gaberscek, M.-L. Doublet. Origin of the 
voltage hysteresis in the CoP conversion material for Li-ion batteries. The Journal of Physical Chemistry C 117 (2013) 837-849.

41. M.A. Roscher, D.U. Sauer. Dynamic electric behavior and open-circuit-voltage modeling of $\mathrm{LiFePO}_{4}$-based lithium ion secondary batteries. Journal of Power Sources 196 (2011) 331-336.

42. W.J. Zhang. Lithium insertion/extraction mechanism in alloy anodes for lithium-ion batteries. Journal of Power Sources 196 (2011) 877-885.

43. A. K. Sleigh, J. J. Murray, W. R. Mckinnon. Memory effects due to phase conversion and hysteresis in $\mathrm{Li} / \mathrm{Li}_{\mathrm{x}} \mathrm{MnO}_{2}$ cells. Electrochimica Acta 36 (1991) 1469-1474.

44. V. Srinivasan, J. Newman. Existence of path-dependence in the $\mathrm{LiFePO}_{4}$ electrode. Electrochemical and Solid-State Letters 9 (2006) A110-A114.

45. J.P. Christophersen, G.L. Hunt, C.D. Ho, et al. Pulse resistance effects due to charging or discharging of high-power lithium-ion cells: A path dependence study. Journal of Power Sources 173 (2007) 998-1005.

46. V. Srinivasan, J. Newman. Discharge model for the lithium iron-phosphate electrode. Journal of the Electrochemical Society 151 (2004) A1517-A1529.

47. C. Delmas, M. Maccario, L. Croguennec, et al. Lithium deintercalation in $\mathrm{LiFePO}_{4}$ nanoparticles via a domino-cascade model. Nature Materials 7 (2008) 665-671.

48. W. Dreyer, C. Guhlke, R. Huth. The behavior of a many-particle electrode in a lithium-ion battery. Physica D 240 (2011) 1008-1019.

49. J. Cannarella, C.B. Arnold. State of health and charge measurements in lithium-ion batteries using mechanical stress. Journal of Power Sources 269 (2014) 7-14. 
50. M. Dubarry, C. Truchot, B.Y. Liaw, K. Gering, S. Sazhin, D. Jamison, C. Michelbacher. Evaluation of commercial lithium-ion cells based on composite positive electrode for plug-in hybrid electric vehicle applications III. Effect of thermal excursions without prolonged thermal aging. Journal of the Electrochemical Society 160 (2013) A191-A199.

51. M. Dubarry, C. Truchot, B.Y. Liaw, K. Gering, S. Sazhin, D. Jamison, C. Michelbacher. Evaluation of commercial lithium-ion cells based on composite positive electrode for plug-in hybrid electric vehicle applications. Part II. Degradation mechanism under 2C cycle aging. Journal of Power Sources 196 (2011) 10336-10343.

52. Y. Wang, C. Zhang, Z. Chen. A method for joint estimation of state-of-charge and available energy of $\mathrm{LiFePO}_{4}$ batteries. Applied Energy 135 (2014) 81-87.

53. Y. Hua, A. Cordoba-Arenas, N. Warner, G. Rizzoni. A multi time-scale state-of-charge and state-of-health estimation framework using nonlinear predictive filter for lithium-ion battery pack with passive balance control. Journal of Power Sources 280 (2015) 293-312.

54. G. Liu, M. Ouyang, L. Lu, J. Li, X. Han. Online estimation of lithium-ion battery remaining discharge capacity through differential voltage analysis. Journal of Power Sources 274 (2015) 971-989.

55. F. Sun, R. Xiong, H. He. Estimation of state-of-charge and state-of-power capability of lithium-ion battery considering varying health conditions. Journal of Power Sources 259 (2014) 166-176.

56. Y. Zou, X. Hu, H. Ma, S.E. Li. Combined state of charge and state of health estimation 
over lithium-ion battery cell cycle lifespan for electric vehicles. Journal of Power Sources 273 (2015) 793-803.

57. S.L. Wu, H.C. Chen, S.R. Chou. Fast estimation of state of charge for lithium-ion batteries. Energies 7 (2014) 3438-3452.

58. M.H. Hung, C.H. Lin, L.C. Lee, C.M. Wang. State-of-charge and state-of-health estimation for lithium-ion batteries based on dynamic impedance technique. Journal of Power Sources 268 (2014) 861-873.

59. F. Leng, C.M. Tan, R. Yazami, M.D. Le. A practical framework of electrical based online state-of-charge estimation of lithium ion batteries. Journal of Power Sources 255 (2014) 423-430.

60. C. Zhang, J. Jiang, W. Zhang, Y. Wang, S.M. Sharkh, R. Xiong. A novel data-driven fast capacity estimation of spent electric vehicle lithium-ion batteries. Energies 7 (2014) 8076-8094.

61. F. Sun and R. Xiong. A novel dual-scale cell state-of-charge estimation approach for series-connected battery pack used in electric vehicles. Journal of Power Sources 274 (2015) 582-594.

62. H. Dai, P. Guo, X. Wei, Z. Sun, J. Wang. ANFIS (adaptive neuro-fuzzy inference system) based online SOC (State of Charge) correction considering cell divergence for the EV (electric vehicle) traction batteries. Energy 80 (2015) 350-360.

63. C. Weng, J. Sun and H. Peng. A unified open-circuit-voltage model of lithium-ion batteries for state-of-charge estimation and state-of-health monitoring. Journal of Power Sources 258 (2014) 228-237. 
64. F. Feng, R. Lu and C. Zhu. A combined state of charge estimation method for lithium-ion batteries used in a wide ambient temperature range. Energies 7 (2014) 3004-3032.

65. M. García-Plaza, D. Serrano-Jiménez, J. Eloy-García Carrasco, J. Alonso-Martínez. A $\mathrm{Ni}-\mathrm{Cd}$ battery model considering state of charge and hysteresis effects. Journal of Power Sources 275 (2015) 595-604.

66. H. Chaoui, N. Golbon, I. Hmouz, R. Souissi, S. Tahar. Lyapunov-based adaptive state of charge and state of health estimation for lithium-ion batteries. IEEE Transactions on Industrial Electronics 62 (2015) 1610-1618.

67. C.Y. Chun, J. Baek, G.S. Seo, B.H. Cho, J. Kim, I. Chang, S. Lee. Current sensor-less state-of-charge estimation algorithm for lithium-ion batteries utilizing filtered terminal voltage. Journal of Power Sources 273 (2015) 255-263.

68. W. Wang, H. Shu-Hung Chung, J. Zhang. Near-real-time parameter estimation of an electrical battery model with multiple time constants and SOC-dependent capacitance. IEEE Transactions on Power Electronics 29 (2014) 5905-5920.

69. N. Yang, X. Zhang, G. Li. State of charge estimation for pulse discharge of a $\mathrm{LiFePO}_{4}$ battery by a revised Ah counting. Electrochimica Acta 151 (2015) 63-71.

70. N. Yang, X. Zhang, G. Li. State-of-charge estimation for lithium ion batteries via the simulation of lithium distribution in the electrode particles. Journal of Power Sources 272 (2014) 68-78.

71. M. Ouyang, G. Liu, L. Lu, J. Li, X. Han. Enhancing the estimation accuracy in low state-of-charge area: A novel onboard battery model through surface state of charge 
determination. Journal of Power Sources 270 (2014) 221-237.

72. F. Zhong, H. Li, S. Zhong, Q. Zhong, C. Yin. An SOC estimation approach based on adaptive sliding mode observer and fractional order equivalent circuit model for lithium-ion batteries. Communications in Nonlinear Science and Numerical Simulation 24 (2015) 127-144.

73. A. Alfi, M. Charkhgard, M.H. Zarif. Hybrid state of charge estimation for lithium-ion batteries: design and implementation. IET Power Electronics 7 (2014) 2758-2764.

74. R. Ahmed, M. El Sayed, I. Arasaratnam, T. Jimi, S. Habibi. Reduced-order electrochemical model parameters identification and SOC estimation for healthy and aged Li-ion batteries Part I: Parameterization model development for healthy batteries. IEEE Journal of Emerging and Selected Topics in Power Electronics 2 (2014) 659-677.

75. R. Ahmed, M. El Sayed, I. Arasaratnam, T. Jimi, S. Habibi. Reduced-order electrochemical model parameters identification and state of charge estimation for healthy and aged Li-ion batteries; Part II: Aged battery model and state of charge estimation. IEEE Journal of Emerging and Selected Topics in Power Electronics 2 (2014) 678-690.

76. B. Balasingam, G.V. Avvari, B. Pattipati, K.R. Pattipati, Y. Bar-Shalom. A robust approach to battery fuel gauging, part II: Real time capacity estimation. Journal of Power Sources 269 (2014) 949-961.

77. C. Burgos, D. Sáez, M.E. Orchard, R. Cárdenas. Fuzzy modelling for the state-of-charge estimation of lead-acid batteries. Journal of Power Sources 274 (2015) 355-366. 
78. J. Du, Z. Liu, Y. Wang. State of charge estimation for Li-ion battery based on model from extreme learning machine. Control Engineering Practice 26 (2014) 11-19.

79. H. Fang, X. Zhao, Y. Wang, Z. Sahinoglu, T. Wada, S. Hara, R.A. de Callafon. Improved adaptive state-of-charge estimation for batteries using a multi-model approach. Journal of Power Sources 254 (2014) 258-267.

80. X. Han, M. Ouyang, L. Lu, J. Li. Simplification of physics-based electrochemical model for lithium ion battery on electric vehicle. Part II: Pseudo-two-dimensional model simplification and state of charge estimation. Journal of Power Sources 278 (2015) 814-825.

81. J.N. Hu, J.J. Hu, H.B. Lin, X.P. Li, C.L. Jiang, X.H. Qiu, W.S. Li. State-of-charge estimation for battery management system using optimized support vector machine for regression. Journal of Power Sources 269 (2014) 682-693.

82. L. Kang, X. Zhao, J. Ma. A new neural network model for the state-of-charge estimation in the battery degradation process. Applied Energy 121 (2014) 20-27.

83. D. Li, J. Ouyang, H. Li, J. Wan. State of charge estimation for $\mathrm{LiMn}_{2} \mathrm{O}_{4}$ power battery based on strong tracking sigma point Kalman filter. Journal of Power Sources 279 (2015) 439-449.

84. X. Liu, Z. Chen, C. Zhang, J. Wu. A novel temperature-compensated model for power Li-ion batteries with dual-particle-filter state of charge estimation. Applied Energy 123 (2014) 263-272.

85. H. Rahimi-Eichi, F. Baronti, M. Chow. Online adaptive parameter identification and state-of-charge coestimation for lithium-polymer battery cells. IEEE Transactions on 
Industrial Electronics 61 (2014) 2053-2061.

86. Y. Tian, C. Chen, B. Xia, W. Sun, Z. Xu, W. Zheng. An adaptive gain nonlinear observer for state of charge estimation of lithium-ion batteries in electric vehicles. Energies 7 (2014) 5995-6012.

87. Y. Tian, B. Xia, W. Sun, Z. Xu, W. Zheng. A modified model based state of charge estimation of power lithium-ion batteries using unscented Kalman filter. Journal of Power Sources 270 (2014) 619-626.

88. Y. Tian, B. Xia, M. Wang, W. Sun, Z. Xu. Comparison study on two model-based adaptive algorithms for SOC estimation of lithium-ion batteries in electric vehicles. Energies 7 (2014) 8446-8464.

89. C. Unterrieder, C. Zhang, M. Lunglmayr, R. Priewasser, S. Marsili, M. Huemer. Battery state-of-charge estimation using approximate least squares. Journal of Power Sources 278 (2015) 274-286.

90. T.T. Vo, X. Chen, W. Shen, A. Kapoor. New charging strategy for lithium-ion batteries based on the integration of Taguchi method and state of charge estimation. Journal of Power Sources 273 (2015) 413-422.

91. Y. Wang, C. Zhang, Z. Chen. A method for state-of-charge estimation of Li-ion batteries based on multi-model switching strategy. Applied Energy 137 (2015) 427-434.

92. Y. Wang, C. Zhang, Z. Chen. A method for state-of-charge estimation of $\mathrm{LiFePO}_{4}$ batteries at dynamic currents and temperatures using particle filter. Journal of Power Sources 279 (2015) 306-311.

93. B. Xia, C. Chen, Y. Tian, W. Sun, Z. Xu, W. Zheng. A novel method for state of charge 
estimation of lithium-ion batteries using a nonlinear observer. Journal of Power Sources 270 (2014) 359-366.

94. J. Xu, B. Cao, Z. Chen, Z. Zou. An online state of charge estimation method with reduced prior battery testing information. International Journal of Electrical Power \& Energy Systems 63 (2014) 178-184.

95. J. Xu, C.C. Mi, B. Cao, J. Deng, Z. Chen, S. Li. The state of charge estimation of lithium-ion batteries based on a proportional-integral observer. IEEE Transactions on Vehicular Technology 63 (2014) 1614-1621.

96. X. Hu, F. Sun, Y. Zou. Estimation of state of charge of a lithium-ion battery pack for electric vehicles using an adaptive Luenberger observer. Energies 3 (2010) 1586-1603.

97. J.P. Schmidt, P. Berg, M. Schönleber, A. Weber, E. Ivers-Tiffée. The distribution of relaxation times as basis for generalized time-domain models for $\mathrm{Li}$-ion batteries. Journal of Power Sources 221 (2013) 70-77.

98. T.K. Dong, A. Kirchev, F. Mattera, J. Kowal, Y. Bultel. Dynamic modeling of Li-ion batteries using an equivalent electrical circuit. Journal of the Electrochemical Society 158 (2011) A326-A336.

99. P.L. Moss, G. Au, E.J. Plichta, J.P. Zheng. An electrical circuit for modeling the dynamic response of li-ion polymer batteries. Journal of The Electrochemical Society 155 (2008) A986-A994.

100. J.M. Francisco, J. Sabatier, L. Lavigne, F. Guillemard, M. Moze, M. Tari, M. Merveillaut, A. Noury. Lithium-ion battery state of charge estimation using a fractional battery model. International Conference on Fractional Differentiation and Its 
Applications (ICFDA), 2014. IEEE, (2014) 1-6.

101. J. Sabatier, J.M. Francisco, F. Guillemard, L. Lavigne, M. Moze, M. Merveillaut. Lithium-ion batteries modeling: A simple fractional differentiation based model and its associated parameters estimation method. Signal Processing 107 (2015) 290-301.

102. M. Dubarry, B.Y. Liaw. Identify capacity fading mechanism in a commercial $\mathrm{LiFePO}_{4}$ cell. Journal of Power Sources 194 (2009) 541-549.

103. Z. Li, J. Zhang, B. Wu, J. Huang, Z. Nie, Y. Sun, F. An, N. Wu. Examining temporal and spatial variations of internal temperature in large-format laminated battery with embedded thermocouples. Journal of Power Sources 241 (2013) 536-553.

104. J. Zhang, B. Wu, Z. Li, J. Huang. Simultaneous estimation of thermal parameters for large-format laminated lithium-ion batteries. Journal of Power Sources 259 (2014) 106-116.

105. B. Wu, Z. Li, J. Zhang. Thermal design for the pouch-type large-format lithium-ion batteries. I. Thermo-electrical modeling and origins of temperature non-uniformity. Journal of The Electrochemical Society 162 (2015) A181-A191.

106. M. Doyle, T.F. Fuller, and J. Newman. Modeling of galvanostatic charge and discharge of the lithium/polymer/insertion cell. Journal of the Electrochemical Society 140 (1993) $1526-1533$.

107. J. Newman, K.E. Thomas, H. Hafezi, D.R. Wheeler. Modeling of lithium-ion batteries. Journal of Power Sources 119 (2003) 838 -843.

108. P. Arora, M. Doyle, R.E. White. Mathematical modeling of the lithium deposition overcharge reaction in lithium-ion batteries using carbon-based negative electrodes. $J$. 
Electrochem. Soc. 146 (1999) 3543-3553.

109. M. Tang, P. Albertus, J. Newman. Two-dimensional modeling of lithium deposition during cell charging. J. Electrochem. Soc. 156 (2009) A390-A399.

110. R.D. Perkins, A.V. Randll, X. Zhang, G.L. Plett. Controls oriented reduced order modeling of lithium deposition on overcharge. J. Power Sources 209 (2012) 318-325.

111. A. Jossen. Fundamentals of battery dynamics. Journal of Power Sources 154 (2006) $530-538$.

112. K.A. Smith, C.D. Rahn, C. Wang. Control oriented 1D electrochemical model of lithium ion battery. Energy Conversion and Management 48 (2007) 2565-2578.

113. H. Maleki, S. Al Hallaj, J.R. Selman, R.B. Dinwiddie, H. Wang. Thermal properties of lithium-ion battery and components. Journal of the Electrochemical Society 146 (1999) 947-954.

114. S.A. Hallaj, H. Maleki, J.S. Hong, J.R. Selman. Thermal modeling and design considerations of lithium-ion batteries. Journal of Power Sources 83 (1999) 1-8.

115. S.A. Hallaj, J.R. Selman. Thermal modeling of secondary lithium batteries for electric vehicle/hybrid electric vehicle applications. Journal of Power Sources 110 (2002) 341-348.

116. S.E. Gustafsson. Transient plane source techniques for thermal-conductivity and thermal-diffusivity measurements of solid materials. Review of Scientific Instruments $62(1991) 797-804$.

117. H. Yi. Rapid thermal conductivity measurement with a hot disk sensor - Part 1. Theoretical considerations. Thermochimica Acta 436 (2005) 122-129. 
118. H. Yi. Rapid thermal conductivity measurement with a hot disk sensor - Part 2. Characterization of thermal greases. Thermochimica Acta 436 (2005) 130-134.

119. K. Onda, T. Ohshima, M. Nakayama, K. Fukuda, T. Araki. Thermal behavior of small lithium-ion battery during rapid charge and discharge cycles. Journal of Power Sources 158 (2006) 535-542.

120. D.W. Dees, V.S. Battaglia, A. Belanger. Electrochemical modeling of lithium polymer batteries. Journal of Power Sources 110 (2002) 310-320.

121. A. Pesaran. Electrothermal analysis of lithium ion batteries. The 23rd International Battery Seminar \& Exhibit, March 2006, Florida, USA.

122. M.S. Wu, K.H. Liu, Y.Y. Wang, C.C. Wan. Heat dissipation design for lithium-ion batteries. Journal of Power Sources 109 (2002) 160-166.

123. S.C. Chen, Y.Y. Wang, C.C. Wan. Thermal analysis of spirally wound lithium batteries. Journal of The Electrochemical Society 153 (2006) A637-A648.

124. K.H. Kwon, C.B. Shin, T.H. Kang, C.S. Kim. A two-dimensional modeling of a lithium-polymer battery. Journal of Power Sources 163 (2006) 151-157.

125. U.S. Kim, C.B. Shin, C.S. Kim. Modeling for the scale-up of a lithium-ion polymer battery. Journal of Power Sources 189 (2009) 841-846.

126. Y. Inui, Y. Kobayashi, Y. Watanabe, Y. Watase, Y. Kitamura. Simulation of temperature distribution in cylindrical and prismatic lithium ion secondary batteries. Energy Conversion and Management 48 (2007) 2103-2109.

127. Y.F. Chen, J.W. Evans. Three-dimensional thermal modeling of lithium-polymer batteries under galvanostatic discharge and dynamic power profile. J. Electrochem. Soc. 
141 (1994) 2947-2955.

128. Y.F. Chen, J.W. Evans. Thermal analysis of lithium-ion batteries. J. Electrochem. Soc. 143 (1996) 2708-2712.

129. S.C. Chen, C.C. Wan, Y.Y. Wang. Thermal analysis of lithium-ion batteries. Journal of Power Sources 140 (2005) 111-124.

130. R.R. Richardson, P.T. Ireland, D.A. Howey. Battery internal temperature estimation by combined impedance and surface temperature measurement. Journal of Power Sources 265 (2014) 254-261.

131. A. Hande. Internal battery temperature estimation using series battery resistance measurements during cold temperatures. Journal of Power Sources 158 (2006) 1039-1046.

132. H. Dai, L. Zhu, J. Zhu, X. Wei, Z. Sun. Adaptive Kalman filtering based internal temperature estimation with an equivalent electrical network thermal model for hard-cased batteries. Journal of Power Sources 293 (2015) 351-365.

133. L. Jasinski. Rapid battery charging system and method. U.S. Patent 3,852,652, Dec 3, 1974.

134. C. Forgez, D.V. Do, G. Friedrich, M. Morcrette, C. Delacourt. Thermal modeling of a cylindrical $\mathrm{LiFePO}_{4} /$ graphite lithium-ion battery. Journal of Power Sources 195 (2010) 2961-2968.

135. C.Y. Lee, S.J. Lee, M.S. Tang, P.C. Chen. In situ monitoring of temperature inside lithium-ion batteries by flexible micro temperature sensors. Sensors 11 (2011) 9942-9950. 
136. C.Y. Lee, F.B. Weng, C.K. Cheng, C.P. Chang. In situ monitoring of high-temperature proton exchange membrane fuel cell stack using flexible micro temperature and voltage sensors. Journal of Power Sources 205 (2012) 345-349.

137. G. Zhang, L. Cao, S. Ge, C.Y. Wang, C.E. Shaffer, C.D. Rahn. In situ measurement of Li-ion battery internal temperature. Abstract \#538, the 224th ECS Meeting, 2013, The Electrochemical Society.

138. G. Zhang, L. Cao, S. Ge, C.Y. Wang, C.E. Shaffer, C.D. Rahn. In situ measurement of radial temperature distributions in cylindrical Li-ion cells. Journal of The Electrochemical Society 161 (2014) A1499-A1507.

139. M. Ouyang, D. Ren, L. Lu, J. Li, X. Feng, X. Han, G. Liu. Overcharge-induced capacity fading analysis for large format lithium-ion batteries with $\mathrm{Li}_{\mathrm{y}} \mathrm{Ni}_{1 / 3} \mathrm{Co}_{1 / 3} \mathrm{Mn}_{1 / 3} \mathrm{O}_{2}+\mathrm{Li}_{\mathrm{y}} \mathrm{Mn}_{2} \mathrm{O}_{4}$ composite cathode. Journal of Power Sources 279 (2015) 626-635

140. M. Ouyang, J. Li, F. Yang, L. Lu. Automotive new powertrains: systems, models, and controls, Tsinghua University Press, 2008.

141. J. Zhang, H. Ge, Z. Li. Internal heating of lithium-ion batteries using alternating current based on the heat generation model in frequency domain. Journal of Power Sources 273 (2015) 1030-1037.

142. D. Bernardi, E. Pawlikowski, J. Newman. A general energy balance for battery systems. J. Electrochem. Soc. 132 (1985) 5-12.

143. J. Huang, Z. Li, J. Zhang, S. Song, Z. Lou, N. Wu. An analytical three-scale impedance model for porous electrode with agglomerates in lithium-ion batteries. Journal of The 
Electrochemical Society 162 (2015) A585- A595.

144. M. Dubarry, N. Vuillaume, B.Y. Liaw. Origins and accommodation of cell variations in Li-ion battery pack modeling. Int. J. Energy Res. 34 (2010) 216-231.

145. M. Dubarry, C. Truchot, A. Devie, B.Y. Liaw. State-of-charge determination in lithium-ion battery packs based on two-point measurements in life. J. Electrochem. Soc. 162 (2015) A877-A884.

146. B. Sun, J. Jiang, F. Zheng, W. Zhao, B.Y. Liaw, H. Ruan, Z. Han, W. Zhang. Practical state of health estimation of power batteries based on Delphi method and grey relational grade analysis. J. Power Sources 282 (2015) 146-157. 


\section{FIGURE CAPTIONS}

Figure 1. A logic tree of SOC-related subjects discussed in this work.

Figure 2. Schematic of a first-order ECM for illustration.

Figure 3. A typical EIS of a lithium-ion cell in a Nyquist plot

Figure 4. The thermal time constants of a laminated cell in an external heating experiment reported in Ref. [103].

Figure 5. Time constant span of electrical, thermal and mechanical properties in a cell.

Figure 6. Important aspects in the coupling of electrical, thermal and mechanical properties.

Figure 7. DC resistance (DCR) and specific power curve of a $22 \mathrm{Ah}$ graphite- $\mathrm{LiFePO} 4$ lithium-ion battery [34].

Figure 8. Distributions of (a) DCR, (b) end-of-charge (EOC) current and rest cell voltage (RCV), and capacity at (c) $\mathrm{C} / 2$ and (d) $\mathrm{C} / 5$ in a batch of 100 commercial cells [144].

\section{TABLE CAPTIONS}

Table 1. Experimental results describing the characteristics of the hysteresis phenomenon.

Table 2. Main mechanistic models related to the hysteresis phenomenon.

Table 3. Approaches to use electrochemical impedance to calibrate empirical SOC.

Table 4. Remaining issues of using mechanical stress to calibrate empirical SOC.

Table 5. Three basic physical properties of a lithium-ion cell.

Table 6. The geometry and the pre-determined thermal properties of the cell in Ref. [104].

Table 7. Comparison of the requirements in parameter identification in two types of models. 
Table 8. Dimension selection of thermal models for batteries of different structures.

Table 9. Issues in thermal measurements and possible solutions.

Table 10. Solutions to minimize validation insufficiency. 
Table 1.

\begin{tabular}{|c|l|l|}
\hline No. & \multicolumn{1}{|c|}{ Characteristics } & \multicolumn{1}{c|}{ Details } \\
\hline 1 & Temporally stable & $\begin{array}{l}\text { Hysteretic voltage gap will not change with extra rest time and eventually diminish, which is } \\
\text { the main basis for distinguishing hysteresis and equalization. }\end{array}$ \\
\hline 2 & Irrelevant to C-rate & Hysteresis voltage gap is irrelevant to the C-rate of preceding charging or discharging. \\
\hline 3 & $\begin{array}{l}\text { Enveloped by the } \\
\text { primary hysteresis } \\
\text { curve [37] }\end{array}$ & $\begin{array}{l}\text { The outline of the hysteresis voltage gap in the entire SOC range constitutes the primary } \\
\text { hysteresis curve, and that in a portion of the SOC range forms a secondary curve. All the pieces } \\
\text { of secondary curves are enveloped in the primary one. }\end{array}$ \\
\hline
\end{tabular}


Table 2.

\begin{tabular}{|c|l|l|}
\hline No. & \multicolumn{1}{|c|}{ Models } & \multicolumn{1}{c|}{ Details } \\
\hline 1 & $\begin{array}{l}\text { Single-particle } \\
\text { theory }[44,46]\end{array}$ & $\begin{array}{l}\text { Taking the phase transformation process of } \mathrm{FePO}_{4} / \mathrm{LiFePO}_{4} \text { as an example, the front of phase } \\
\text { transformation gradually advances in the radial direction of each single particle. Assuming that } \\
\text { the phase transitions of different particles are very similar, studying the phase transformation } \\
\text { process of a single particle could reveal the main information of battery phase transformation. }\end{array}$ \\
\hline 2 & $\begin{array}{l}\text { Multi-particle } \\
\text { domain theory }[47- \\
48]\end{array}$ & $\begin{array}{l}\text { Battery phase transformation process should be described as multi-particle domains as the } \\
\text { object. In the lithium intercalation process, the two-phase interface advances in the multi- } \\
\text { particle domains with its front in a particular shape. Individual particles behind the interface are } \\
\text { in lithium-rich state while particles expecting this interface front are lithium-depleted. In any } \\
\text { single particle, its internal lithium-ion distribution is uniform. }\end{array}$ \\
\hline
\end{tabular}


Table 3.

\begin{tabular}{|c|l|l|l|}
\hline No. & \multicolumn{1}{|c|}{ Approach } & \multicolumn{1}{|c|}{ Approach details } & \multicolumn{1}{c|}{ Drawbacks } \\
\hline 1 & Frequency scan & $\begin{array}{l}\text { Measure the impedance over a wide frequency } \\
\text { range to recognize each impedance elements, and } \\
\text { then use one or several specific elements } \\
\text { (resistance, capacitance, time constant, etc.) to } \\
\text { determine SOC }\end{array}$ & $\begin{array}{l}\text { Presence of multiple ECD models } \\
\text { could impair the component } \\
\text { recognition. }\end{array}$ \\
\hline 2 & Single frequency & $\begin{array}{l}\text { Correlate the amplitude or the phase angle of } \\
\text { impedance at single-frequency with SOC }\end{array}$ & $\begin{array}{l}\text { To identify which frequency is } \\
\text { informative with respect to SOC }\end{array}$ \\
\hline
\end{tabular}


Table 4.

\begin{tabular}{|c|l|}
\hline No. & \multicolumn{1}{|c|}{ Emerging issues with mechanical stress to calibrate SOC } \\
\hline 1 & $\begin{array}{l}\text { Whereas measuring the thickness accounts for only the scalar factor of the normal stress, and assuming the sum of the shear stress is } \\
\text { zero, mechanical stress is a force that should be expressed in a vector, depending on the force field distribution in the electrode matrix. } \\
\text { For example, not only the assumption of zero sums in shear stress might not be true in certain electrode designs and aspect ratios, but } \\
\text { also the secondary effect of shear stress on the neighboring compositions might not be uniform if the deformation is direction } \\
\text { dependent. }\end{array}$ \\
\hline 2 & $\begin{array}{l}\text { Chemical, electrical and thermal balances in the electrode matrix and the entire chemical system in the cell could affect stress-strain field } \\
\text { balance. Such balance is spatial and time dependent. During the cell balance, the dynamic behavior of the surface stress as a function of } \\
\text { spatial location and time is not well defined in the transient state. This could introduce significant errors in the initial stage of balance } \\
\text { during stress or strain measurements. }\end{array}$ \\
\hline 3 & $\begin{array}{l}\text { As explained above, the correlation between the mechanical stress and SOC is also affected by the external conditions, such as the } \\
\text { temperature distribution, the mechanical property of the plates (e.g. thickness, strength, toughness, hardness and plasticity of the } \\
\text { plates), aspect ratio, etc. }\end{array}$ \\
\hline 4 & $\begin{array}{l}\text { Due to the porous nature of the electrode architecture and composite nature of the solid-liquid matrix, the stress-strain field could cause } \\
\text { mass flows of solid and liquid redistributions to alleviate the force balances in the matrix. Over time, accumulative plastic flow may lead } \\
\text { to shape change, as a result of mass redistribution, which causes permanent or semi-permanent (e.g. a longer time lag versus } \\
\text { measurement interval) variations in the thickness and roughness, thus poor consistency in the mechanical stress vs. SOC correlation. }\end{array}$ \\
\hline
\end{tabular}


Table 5

\begin{tabular}{|c|c|c|c|c|c|}
\hline No. & Property & Input & Output & Model \\
\hline 1 & Electrical & Current & Terminal voltage & $\begin{array}{c}\text { ECM/Electrochemical } \\
\text { model }\end{array}$ & See Table 8 for detail \\
\hline 2 & Thermal & $\begin{array}{c}\text { Ambient } \\
\text { temperatur } \\
\text { e }\end{array}$ & $\begin{array}{c}\text { Temperature distribution Note 3 } \\
\text { Thermal balancing } \\
\text { model (heat generation- } \\
\text { conductivity- } \\
\text { dissipation) }\end{array}$ & $\begin{array}{c}\text { Heat generation rate, heat conductivity } \\
\text { cyeat convection coefficient }\end{array}$ \\
\hline 3 & Mechanical & $\begin{array}{c}\text { Historical } \\
\text { conditions }\end{array}$ & $\begin{array}{c}\text { Volume change (free state), stress } \\
\text { change (clamped), external } \\
\text { characteristics change: capacity, } \\
\text { resistance or impedance }\end{array}$ & $\begin{array}{c}\text { Elastic modulus, side reaction } \\
\text { parameters }\end{array}$ \\
\hline
\end{tabular}

Note 3: It supposes a scenario of heating the battery by external heat sources. Another possible scenario is, during the charging or discharging of battery, the input and output could switch to the C-rate and the temperature of battery respectively. In the latter situation, the heat source is internal and the heat is conducted and dissipated towards outside. External and internal heating are normally two reverse processes of the heat flux. 
Table 6.

\begin{tabular}{|c|c|c|c|c|}
\hline Symbol & $L$ & $k$ & $\rho$ & $C_{\mathrm{p}}$ \\
\hline Meaning & $\begin{array}{c}\text { Half the thickness of the } \\
\text { laminated cell }\end{array}$ & $\begin{array}{c}\text { Thermal conductivity in the } \\
\text { through-plane direction }\end{array}$ & $\begin{array}{c}\text { Battery } \\
\text { thermal } \\
\text { capacitance }\end{array}$ \\
\hline Unit & $\mathrm{M}$ & $\frac{\mathrm{W}}{\mathrm{m} \cdot \mathrm{K}}$ & $\frac{\mathrm{kg}}{\mathrm{m}^{3}}$ & $\frac{\mathrm{J}}{\mathrm{kg} \cdot \mathrm{K}}$ \\
\hline Value & $3.6 \times 10^{-3}$ & 0.48 & 2300 & 1243 \\
\hline
\end{tabular}


Table 7

\begin{tabular}{|c|c|c|c|}
\hline No. & Item & Electrochemical model & ECM \\
\hline 1 & Parameter types & $\begin{array}{l}\text { Reaction dynamics: electro-conductibility, ion diffusion coefficient, exchange } \\
\text { current density } \\
\text { Geometry: particle size, separator pore fraction, material thickness, electrolyte } \\
\text { concentration, material constitutions (\%) } \\
\text { Equilibrium potential: relation between OCV and ion concentration in solid } \\
\text { material }\end{array}$ & $\begin{array}{l}\qquad R_{\mathrm{ohm}}, R_{\mathrm{SEI}}, R_{\mathrm{ct}} \\
\quad C_{\mathrm{dl}}, W, \text { SOC-OCV curve } \\
\text { ohm: ohmic } \\
\text { SEI: the SEI film } \\
\text { ct: charge transfer } \\
\text { dl: double layer }\end{array}$ \\
\hline 2 & $\begin{array}{c}\text { Number of } \\
\text { parameters in the } \\
\text { model }\end{array}$ & $\sim 50$ & $\sim 10$ \\
\hline 3 & $\begin{array}{l}\text { Identification } \\
\text { experiments }\end{array}$ & $\begin{array}{l}\text { Reaction dynamics : potentiostatic intermittent titration technique (PITT), } \\
\text { galvanostatic intermittent titration technique (GITT), cyclic voltammetry (CV) } \\
\text { and electrochemical impedance spectroscopy (EIS) } \\
\text { Geometry: provided by the manufacturers } \\
\text { Equilibrium potential: OCV-SOC curve, see Section } 2.1 \text { for detail }\end{array}$ & $\begin{array}{c}\text { CC discharging tests under } \\
\text { several rates }\end{array}$ \\
\hline
\end{tabular}


Table 8

\begin{tabular}{|c|c|c|c|}
\hline $\begin{array}{c}\text { Battery } \\
\text { structure }\end{array}$ & One dimension & Two dimensions & Three dimensions \\
\hline \multirow{2}{*}{ Cylindrical } & \multirow{2}{*}{ Radius, r [114,115,119] } & $\begin{array}{c}\text { Radius, } \mathrm{r} \\
\text { Axis, z }[121,122]\end{array}$ & \multirow{2}{*}{$\begin{array}{l}\text { Radius, } \mathrm{r} \\
\text { Axis, z } \\
\text { Angel, } \theta\end{array}$} \\
\hline & & $\begin{array}{c}\text { Radius, } r \\
\text { Angel, } \theta \text { [123] }\end{array}$ & \\
\hline Prismatic & Axis, $\mathrm{z}$ & $\begin{array}{l}\text { Long radius, } \mathrm{x} \\
\text { Short radius, } \mathrm{y}\end{array}$ & $\begin{array}{l}\text { Long radius, } \mathrm{x} \\
\text { Short radius, y } \\
\text { Axis, z [126] }\end{array}$ \\
\hline Laminated & Thickness / through-plane, y [120] & $\begin{array}{c}\text { Length, } \mathrm{x} \\
\text { Height, } \mathrm{z}[124,125]\end{array}$ & $\begin{array}{c}\text { Length, } \mathrm{x} \\
\text { Thickness, } \mathrm{y} \\
\text { Height, z [127-129] }\end{array}$ \\
\hline
\end{tabular}


Table 9

\begin{tabular}{|c|c|c|c|}
\hline \multirow{2}{*}{ No. } & Sensor type & Error origin & Solution \\
\hline \multirow{3}{*}{1} & \multirow{2}{*}{\begin{tabular}{c} 
Infrared thermal imaging \\
\cline { 3 - 4 }
\end{tabular}} & A non-flat surface or surface roughness & $\begin{array}{c}\text { Correction according to the angle of } \\
\text { incidence }\end{array}$ \\
\cline { 3 - 4 } & & Surface emissivity & Surface paint \\
\cline { 3 - 4 } 2 & \multirow{2}{*}{$\begin{array}{c}\text { Thermocouple / thermal } \\
\text { resistance }\end{array}$} & Environment influence & Tests in dark box \\
\cline { 3 - 4 } & & Non-firm contact & Heat conductive silicone grease \\
\hline
\end{tabular}


Table 10

\begin{tabular}{|c|c|c|c|c|}
\hline No. & Type & Insufficiency & Solution & Solution examples \\
\hline \multirow[t]{5}{*}{1} & \multirow[t]{5}{*}{ Temporal } & \multirow[t]{5}{*}{ Single load profile } & Multiple load tests & (1) CC \\
\hline & & & & (2) Pulse \\
\hline & & & & (3) Sinusoidal or rectangular waves \\
\hline & & & & (4) Dynamic \\
\hline & & & Typical load test & $\begin{array}{l}\text { Using special methods to summarize one typical load to } \\
\text { approximate real-road condition. Such methods are like } \\
\text { hierarchical clustering, fuzzy logic, etc. }\end{array}$ \\
\hline \multirow[t]{4}{*}{2} & \multirow[t]{4}{*}{ Spatial } & \multirow{4}{*}{$\begin{array}{l}\text { Single location } \\
\text { measurement }\end{array}$} & Multi-location measurement & Placing thermocouple matrix on the battery surface [103] \\
\hline & & & Bulk measurement & $\begin{array}{l}\text { Using DC resistance or EIS to determine a bulk temperature } \\
\text { [130-132] }\end{array}$ \\
\hline & & & Field measurement & Infrared thermal imaging \\
\hline & & & Internal measurement & $\begin{array}{l}\text { Using internal thermocouples embedded inside cells }[103,133- \\
138]\end{array}$ \\
\hline
\end{tabular}

